\title{
RELATIVITÉS ET PUISSANCES SPECTRALES CHEZ GASTON BACHELARD
}

\author{
Charles ALunNI
}

Résumé : La Valeur inductive de la relativité est sans conteste l'ouvrage le plus méconnu de toute l'œuvre "philosophique" de Gaston Bachelard. Au silence presque total, à l'absence de lectures, ne répondent que des interprétations du «premier genre », appuyées sur un certain oui-dire discursif, mais qui font l'autorité des pseudo-standards. Les positions bachelardiennes sont ici confrontées à La Déduction relativiste d'Émile Meyerson. Le poids de l'analyse portera essentiellement sur un dépl(o)iement du dispositif bachelardien d'induction et de construction. L'appareillage « inductif » doit être pensé sur le modèle électromagnétique, et la position " constructiviste » est toujours liée au travail de synthèse. Enfin, c'est une véritabie métaphysique que semble dès lors devoir engendrer cette "pensée des sciences ".

Mots-ClÉs : épistémologie, métaphysique, relativité, induction, explication, construction, synthèse, Einstein, Émile Meyerson.

ABSTRACT: La Valeur inductive de la relativité is undoubtedly the least known "philosophical " work of Gaston Bachelard. Before an almost total silence, with the absence of readings, the replies are only "first type " interpretations supported by a certain discursive hearsay, but which have the authority of pseudo-standards. The Bachelardian positions are here confronted by La Déduction relativiste of Émile Meyerson. The weight of the analysis will essentially rest upon a display of the Bachelardian device of induction and of construction. The "inductive " preparation must be thought out on the electromagnetic model and the "constructivist" position is always linked to the effort of synthesis. In short, it is a veritable metaphysics which seemingly must generate this «thought of the sciences».

KEYwORDs : epistemology, metaphysics, relativity, induction, explication, construction, synthesis, Einstein, Émile Meyerson. 
Zusammenfassung : La Valeur inductive de la relativité ist ohne Zweifel der am meisten mißverstandene Aspekt im gesamten "philosophischen "Werk von Gaston Bachelard. Dem beinahe vollständigen Schweigen und der nicht erfolgten Lektüre entsprechen nur einige oberflächliche Interpretationen auf der Grundlage eines gewissen diskursiven Hörensagens, worauf die Autoritât der Pseudo-Standards beruht. In diesem Aufsatz werden Bachelards Positionen mit denjenigen aus Émile Meyersons La Déduction relativiste verglichen. Der Schwerpunkt der Analyse liegt im wesentlichen auf der Erklärung von Bachelards Begriffen der Induktion und der Konstruktion. Das « induktive » Rüstzeug muß gedanklich dem elektromagnetischen Modell nachvollzogen werden, während die "konstruktivistische » Position immer mit der Tätigkeit der Synthese verbunden wird. Es ist am Ende eine echte Metaphysik, aus der dieses «Denken der Wissenschaften 》 hervorzugehen scheint.

STICHWÖRTER: Epistemologie, Metaphysik, Relativität, Induktion, Erklärung, Konstruktion, Synthese, Einstein, Émile Meyerson.

RIASSUNTo: La Valeur inductive de la relativité è senza dubbio il testo meno conosciuto di tutta l'opera di Gaston Bachelard. Di fronte al silenzio quasi totale, all'assenza di letture, non si trovano che delle interpretazioni di "primo genere", basate su alcuni "sentiti dire» discorsivi, che assumono l'autorità di pseudostandard. In questo articolo, le posizioni di Bachelard sono confrontate a La Déducton relativiste di Émile Meyerson. L'analisi si appoggerà soprattutto sul dispiegamento del dispositivo dell'induzione $e$ della costruzione in Bachelard. L'apparato " induttivo"va pensato sul modello elettromagnetico, e la posizione "costruttivista » e' sempre legata al lavoro di Sintesi. In conclusione, si tratta di una vera metafisica che sembra a questo punto generare un vero "pensiero della scienza".

PAROLE CHIAVE: epistemologia, metafisical, relatività, induzione, espiegazione, costruzione, sintesi, Einstein, Émile Meyerson.

Élève de Jean-Toussaint Desanti, Charles AluNNI est né en 1951. Membre et secrétaire technique du Groupe de recherches sur l'enseignement de la philosophie, formé à l'École normale supérieure en 1975 autour de Jacques Derrida, il fut directeur de programme au Collège international de philosophie de 1983 à 1989. Cofondateur du Centre d'études et de recherches sur la philosophie italienne, il est actuellement chargé de recherches à la Scuola normale superiore de Pise (Italie), maître de conférences détaché à la rue d'Ulm, où il dirige depuis 1994 le Laboratoire disciplinaire "Pensée des sciences », et secrétaire général du groupe RIENS (Recherches interdisciplinaires sur les Écoles normales supérieures et les classes préparatoires).

Adresse : Laboratoire disciplinaire "Pensée des sciences ", École normale supérieure, 45, rue d'Ulm, F-75230 Paris Cedex 05.

Courrier électronique : alunnic@minitel.net

Internet : http://www.dmi.ens.fr/users/longo/pense-science.html 
À Paulette Destouches-Février et Éric Émery, pour leur générosité. À mon maître Touki.

«L'expérience n'a plus pour rôle que de mesurer nos actes déployés, et les divergences qu'elle peut déceler ne saurait entamer le cristal de nos gestes. »

Gaston BACHELARD, La Valeur inductive de la relativité (1929).

Il est une particularité des grandes œuvres ou des grands livres scientifiques et philosophiques, c'est d'être confrontés aux aléas mondains que commande leur puissance spéculative d'anticipation. En positif apparent, et c'est en partie le cas de Gaston Bachelard, l'accueil du livre ou de l'œuvre peut recevoir l'estampille quasi immédiate de la notoriété, si ce n'est de la vénération, et ce, pour un certain nombre de décennies. Mais, négatif évident, cela à(a?) un certain prix, imposé aujourd'hui par la vulgate ambiante, surtout quand elle se fonde de ne plus en être pour les avoir illusoirement liquidées : c'est le prix de l'oubli, de l'oblitération, de l'occultation succédant au long processus d'obnubilation originel, voire originaire. Comme le précise Gilles Châtelet : «[...] toute grande pensée, si affûtée soit-elle, périt toujours entre les mains de vestales trop zélées ${ }^{1}$. " Nous savons, grâce au grand Karl Marx, que la considération liquidatrice des félidés vient presque toujours, au bout du compte, frapper au coin des plus grandes œuvres de l'humanité. Bachelard, au même titre que Spinoza ou Hegel, est bel et bien devenu, chez nous, un chien crevé !

Á ce propos, voici ce qu'affirmait Roger Cavaillès, dès 1984, dans un numéro spécial de la très intéressante revue italienne Il Protagora, entièrement consacré à « Gaston Bachelard. Bilancio critico di una epistemolo$\operatorname{gia}^{2} \gg$ :

«Le caractère classique des analyses de Gaston Bachelard et l'importance du nouvel esprit épistémologique qu'elles introduisent ne sauraient désormais être contestés. Classique, Bachelard l'est certainement devenu; mais est-il encore actuel après la troisième des grandes révolutions scientifiques $\mathrm{du} \mathrm{xx}^{e}$ siècle, celle que Bateson - dans son ouvrage intitulé Vers une écologie de l'esprit

1. Gilles Châtelet, Vivre et penser comme des porcs. De l'incitation à l'envie et à l'ennui dans les démocraties-marchés, Paris, Exils, 1998, p. 28.

2. [Pour le premier Centenaire de sa naissance. Université de Lecce], XXIV ${ }^{e}$ Année, janv.juin 1984. 
[1972] - qualifiait de “ cybernétique "? Une nouvelle épistémologie, développant les conséquences du thème de l'auto-organisation ${ }^{3}$, semble aujourd'hui, en France tout au moins, occulter quelque peu l'œuvre bachelardienne. Les concepts utilisés (information, rétroaction, structure dissipative...), les problèmes envisagés (équilibres dynamiques, stabilités structurelles, complexification et organisation des systèmes vivants...), les choix philosophiques inspirant la démarche (néo-mécanisme) nous situent manifestement dans une épistémologie non-bachelardienne, dont on pourra d'ailleurs se demander (ceci n'est pas très loin de ressembler à un paradoxe logique) si elle n'entre pas encore dans le cadre de la philosophie bachelardienne du non.

Cette apparente inactualité de Bachelard.sera notre point de départ... Il s'agira précisément d'établir que cette inactualité n'est qu'apparente, et que, sous le silence qui recouvre aujourd'hui cette œuvre, s'affirme encore une pensée qui n'a pas fini de nous éclairer et de nous séduire ${ }^{4}$.»

Il est question ici d'un processus d'obnubilation (impliquant évidemment le spectre solidarisé de la hantise, de l'obsession qui peut s'exprimer jusqu'à la monomanie "modarde " ou "branchée ", et, faisant toujours supplément, de l'obscurcissement); une obnubilation qui entame presque toujours, d'un même geste, l'arrêt interdit (mais jamais avoué comme tel) devant ce que j'appelais la puissance d'anticipation, c'est-à-dire le pouvoir d'effraction des grands livres - ce qui n'est pas à confondre avec l'impossibilité hégélienne de pouvoir "sauter par-dessus son temps ». Tout cela devrait nous paraître évident, à nous philosophes, qui, que je sache, sommes payés pour rappeler, depuis un certain Platon, que les gesticulations et les simagrées de la doxa n'ont d'autre but qu'une volonté populiste d'offusquer ce qui compte vraiment, c'est-à-dire qui compte sans compter, à la pointe affûtée de la pensée. Il est certains livres et nombre d'cuvres qui auront dû attendre leur moment, leur heure, trop riches de leur heurt, de leur choc projectif avec le passé, de leur effet de propulsion : ce sont les grands livres à venir. J'en citerai au moins quatre exemples, présents certes dans leur disponibilité libraire, mais encore à venir; ce sont des ouvrages qui revendiquent toujours explicitement l'ombre portée du grand Euvre bachelardien: Georges Simondon (sans citer ici de titre particulier), Les Idéalités mathématiques de Jean-Toussaint Desanti, Les Anatomies de la pensée d'Alain Prochiantz, et, n'en déplaise 'à sa modestie et à sa réserve philosophiques naturelles, Les Enjeux du mobile de Gilles Châtelet. Je m'en remets d'ailleurs à sa paranoïa non moins naturelle et bienfaisante pour que cela ne l'oblige pas, désormais, à m’épargner. Je dis «bienfai-

3. Référence faite ici à la publication des Actes du colloque de Cerisy-la-Salle, tenu en juin 1981 et intitulé : L'Auto-organisation. De la physique au politique, Paris, Seuil, 1983.

4. Roger Cavarllès, « L'épistémologie de Gaston Bachelard et la révolution cybernétique $»$, Il Protagora, 2, 1984, p. 175-176. 
sante », et en cela je ne fais à nouveau que répéter la leçon bachelardienne : « Il ne suffit point à l'homme d'avoir raison, il faut qu'il ait raison contre quelqu'un », «[...] l'être agit contre la réalité et non plus en s'égalant à la réalité. Les conduites agressives et les mythes cruels sont, tous deux, des fonctions d'attaque, des principes dynamisants. Ils aiguisent l'être ${ }^{5}$. » Voilà de quoi aiguiser une certaine perspicuitas ontologico-politique.

Dans un premier moment, dégrossir donc les choses : ce qui n'est pas un luxe - je le maintiens - face à l'embonpoint bouffi et débordant d'une certaine épistémologie nourrie aux fast-foods de la culture analytique, doublée du vide philosophique et épistémologique d'une certaine sociologie de la science conseillère des princes du moment.

Je laisse, ici encore, la parole à Bachelard, à cette démarche concentrée et purifiée dans les deux mots qu'il prononça lors d'une émission radiophonique (sur Radio-Nancy en 1961) où Denise Laborde l'interrogeait :

« Pour vous, n'est-ce pas, un bon penseur - un bon philosophe - doit être bien enraciné dans sa terre?

- ... ou bien déraciné.

- Après la terre et l'eau, le feu est le troisième élément essentiel. Vous savez ce que dit familièrement le proverbe populaire : "Pour allumer un feu, il faut être philosophe, amoureux et un peu fou ". Qu'en pensez-vous?

- ... ou incendiaire $"$.

Bachelard, qui s'y connaissait en télé-graphes, télé-phones, T.S.F., et télé-communications, et qui s'y colla plus qu'aucun autre philosophe, savait quant à lui ne pas se laisser aller à devenir le lâche et paresseux médium des médias ${ }^{6}$.

5. Gaston BaCHELARD, La Formation de l'esprit scientifique. Contribution à une psychanalyse de la connaissance objective, Paris, Vrin, 1938, p. 245, 247 sq.; ID., Lautréamont, Paris, José Corti, 1939, p. 183; ou encore, ibid., p. 126-127: "Imposer la raison nous paraît une violence insigne, puisque la raison s'impose d'elle-même. Et nous ne pouvons ici nous déprendre d'une idée qui, sous bien des formes, se glisse dans notre esprit : la sévérité est une psychose; c'est, en particulier, la psychose professionnelle du professeur. Elle est plus grave chez le professeur de mathématiques que chez tout autre; car la sévérité en mathématiques est cohérente; on peut en démontrer la nécessité; elle est l'aspect psychologique d'un théorème. Seul, le professeur de mathématiques peut être à la fois sévère et juste. Si le professeur de rhétorique - perdant le bénéfice de la belle et douce relativité de sa culture - est sévère, il est, du même coup, partial. Aussitôt, il devient un professeur automate. »

6. Le 19 mai 1918, après trente-huit mois de tranchées, Bachelard reçoit la croix de guerre et une citation, comme chef du service télégraphique, à l'ordre de la $5^{\mathrm{c}}$ division de cavalerie. Rapportons cette citation qui ne pouvait être que militaire dans son style : « Dans les journées de la fin de mars 1918, pénétré de ses devoirs, insensible au bruit de la bataille, a établi et sans cesse rétabli pendant deux jours et trois nuits des lignes téléphoniques constamment rompues par le feu de l'ennemi, donnant à ses sapeurs un bel exemple de calme, d'opiniâtreté et d'énergie. " Par ailleurs, on ne compte pas moins de cinquante heures d'émissions de radio et de télévision, ainsi que les textes suivants : La Dialectique de la durée, Paris, Presses universitaires de France, 1936, chap. 1II, «Durée et causalité physique », et " Rêverie et radio », $L a$ Nef, févr.-mars 1951. 


\author{
RÉCEPTIONS ET INTERPRÉTATIONS STANOARD \\ DE LA VALEUR INDUCTIVE DE LA RELATIVITÉ
}

Dans ce qui suit, La Valeur inductive de la relativité $e^{7}$ sera d'abord considérée dans le contexte de son émergence, et dans l'espace de sa réception dès 1929. Puis, sa situation dans l'œuvre, ainsi que sa non-postérité apparente seront brièvement évoquées. Enfin, sera analysée son économie

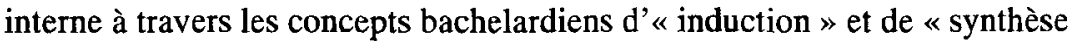
inductive », concepts qui en marquent le titre ${ }^{8}$.

On peut affirmer brutalement que La Valeur inductive de la relativité sera resté l'ouvrage le moins cité - et donc le moins lu - c'est-à-dire le moins commenté, de tous les livres de Bachelard. Lorsque le titre apparaît, c'est simplement dans le cadre d'une liste de rappels bibliographiques ou, tout à fait exceptionnellement, comme illustration vague d'un apparat de notes. Ce texte n'est jamais pris en considération pour lui-même, dans son économie singulière ${ }^{9}$, pas plus que dans sa logique propre. Je prendrai ici l'exemple du bilan établi par Gerald Holton en $1973^{10}$ dans un texte consacré à l'histoire de la théorie de la Relativité : "Biographically or philosophically oriented analyses are also fairly numerous (for example, by

7. G. BAchelard, La Valeur inductive de la relativité, Paris, Vrin, 1929.

8. Enfin, dans une suite que je compte donner au présent article, je dégagerai les notions essentielles de "puissance " et de «virtualité spectrales " que Bachelard tire de ses analyses consacrées aux tenseurs conçus, selon le modèle d'Eddington, comme des "cerveaux tournants $"$.

9. Sur ce constat de rareté des références philosophiques à La Valeur inductive de la relativité, il faut noter une double exception confirmant la règle, celle représentée d'abord par Jean HYPPOLTE que je cite : «La valeur inductive de la Relativité dévoile ce même mouvement de fondation par l'avenir qui anime toute l'épistémologie de G. Bachelard ", dans son texte, "L'épistémologie de G. Bachelard ", Revue d'histoire des sciences, t. XVII, I, janv.mars 1964, p. 8; et celle, encore plus singulière, personnifiée par la physicienne MarieAntoinette Tonnelat - dont le regretté André Lichnerowicz disait d'elle : "Il y a plus de quinze ans [...] [M.-A. Tonnelat] manifestait déjà cet esprit de haute synthèse ", préf. à $L a$ Théorie du champ unifié d'Einstein et quelques-uns de ses développements, Paris, GauthierVillars, 1955, p. vu. Cette insigne relativiste, qui aura résolu explicitement les équations reliant la connexion affine au tenseur fondamental, a, dans ses ouvrages historico-techniques - voir, entre bien d'autres textes, Marie-Antoinette TONNELAT, Histoire du principe de relativité, Paris, Flammarion (Nouvelle Bibliothèque scientifique), 1971, chap. vili, en part., p. 227 $280,357-359,369,462-463$ et 467 -, fait référence systématique à La Valeur inductive de Bachelard. Je tiens ici à remercier Marie Farge pour m'avoir si généreusement donné accès aux manuscrits inédits de l'auteur.

10. Gerald HoLton, Thematic Origins of scientific thought. From Kepler to Einstein, Cambridge, MA, Harvard University Press, 1973. 
Schlick, Reichenbach, Frank, Meyerson, Cassirer, Whitehead, Wenzel, Grünbaum, Polanyi, Margenau, Lenzen, Bridgman, and Northrop) ${ }^{11}$." Notons au passage la brillante absence, aux côtés de Bachelard, d'Arthur Eddington.

Depuis 1929 , on ne connaît que trois recensions en français : une première, anonyme, publiée dans la Revue de métaphysique et de morale ${ }^{12}$, ensuite, deux comptes rendus, l'un de Gaston Rabeau dans la Revue des sciences philosophiques et théologiques ${ }^{13}$; l'autre d'A. Spaier, dans les Recherches philosophiques ${ }^{14}$. Les Recherches venaient en effet, d'être fondées par Alexandre Koyré, H.-Ch. Puech et Spaier, et Bachelard lui-même faisait partie de leur comité. Dès ce premier volume, il publie son texte « Noumène et microphysique » et recense l'ouvrage de Hans Reichenbach, Atom und Kosmos. Das physikalische Weltbild der Gegenwart paru à Berlin en 1930. Walter Dubislav consacre de son côté un article aux Recherches sur la philosophie des mathématiques en Allemagne traduit par Emmanuel Lévinas. De plus, c'est dans ce même volume inaugural, et sous la même rubrique que celle qui chapeaute « Noumène et microphysique » (à savoir «Tendances actuelles de la métaphysique »), qu'est publié le premier texte en français de Martin Heidegger, «De la nature de la cause » (Der Satz vom Grund). Enfin, cela n'est, quant à la rubrique «métaphysique ", qu'une tératologie apparente concernant les positions classiquement attachées à l'œuvre de Bachelard.

Depuis, plus rien, si ce n'est, comme nous allons le voir, un jeu d'opposition et d'effacement face à un autre ouvrage philosophique et épistémologique français reconnu par Albert Einstein lui-même en $1927^{15}$, geste qui procura à son auteur des éloges, à savoir La Déduction relativiste d'Émile Meyerson ${ }^{16}$. L'antagonisme des approches épistémologiques de la Relativité y est aussi radical que l'opposition des titres : déduction versus valeur inductive. Si les zélateurs de l'ouvrage de Meyerson renvoient systématiquement à cette « reconnaissance officielle » de la part d'Einstein, c'est au prix de bien vouloir ignorer deux choses:

a) Le long siège psychologique entrepris par Meyerson auprès d'Einstein dans sa correspondance avec lui ${ }^{17}$. Einstein aura mis pratiquement deux ans pour répondre à la sollicitation pressante de Meyerson. Quant au « style » meyersonien, il suffit de citer ce passage d'une lettre du 20 juillet 1927 :

11. Ibid., p. 11.

12. Suppl. $\mathrm{n}^{\mathrm{0}}$ 3, vol. XXXV, 1928-1929, p.

13. Vol. XVII, 3, 1929, p.

14. Vol. I, 1931, p.

15. Albert Enstein, CEuvres choisies, Paris, Seuil, 1989, vol. IV, « Correspondance française », p. 91 sq.

16. Émile Meyerson, La Déduction relativiste, Paris, Payot, 1925.

17. Ibid., Lettres à Einstein, 20 déc. $1925 ; 28$ mai, 19 juin, 20 juil., 11 oct., 27 oct., 19 déc., 26 déc. 1927. 
«Une dernière prière enfin : dans le dernier paragraphe de votre compte rendu, vous dites de mon livre qu'il est l'un des plus intéressants, etc. Il s'agit là, dans votre bouche, d'un compliment extrêmement élogieux. Mais il semble aussi signifier implicitement que d'autres œuvres philosophiques sont pour ainsi dire ex aequo avec la mienne dans cette compétition. Je ne m'en étais quasiment pas soucié auparavant, notamment pour la raison que ce que je vous avais entendu dire ici, ou ce que m'avaient rapporté certaines personnes de connaissance qui vous avaient parlé, m'inclinait à penser que vous teniez mon travail pour le meilleur (sur le terrain de la théorie de la connaissance). Mais je souhaiterais maintenant beaucoup étudier ces concurrents pour apprendre d'eux. Voudriez-vous me rendre le grand service de m'indiquer des noms et des titres? Je suppose qu'il s'agit au premier chef de Schlick, et je vais naturellement me procurer le livre le plus tôt possible. Mais ne doit-on pas en citer quelque autre ${ }^{18}$ ?»

b) Le fait qu'en 1949, à l'occasion du soixante-dixième anniversaire d'Einstein, le seul représentant français, aux côtés de six prix Nobel scientifiques, n'était autre que Bachelard lui-même ${ }^{19}$. Sur ce point, il faut évoquer ici l'étrange silence de Michel Paty dans sa note de bibliographie ${ }^{20}$, qui renvoie malgré tout à l'ouvrage collectif édité par Arthur P. Schilpp :

«[...] les études d'un auteur sur Einstein peuvent fort bien s'être trouvées en interaction avec la pensée de ce dernier, qui, par exemple y réplique, ou en est influencé. Il suffit d'évoquer les noms de Moritz Schlick, Ernst Cassirer, Hans Reichenbach, ou Émile Meyerson : leurs interprétations de l'œuvre d'Einstein portent la marque de la contemporanéité, et l'auteur qu'ils étudient est en vérité lui-même partie prenante des débats philosophiques sur la Relativité, sur les quanta, et d'une manière générale sur les conceptions de la physique, auxquels contribuent leurs analyses. L'un des derniers ouvrages de ce genre paru du vivant d'Einstein, Albert Einstein philosopher-scientist [...], s'avère même être à cet égard l'un des plus importants, suscitant son texte épistémologique fondamental, la " Réponse aux critiques" [...] »

Sur ce partage inégal des reconnaissances, et sur ses conséquences épistémologiques - ce qui évidemment est la seule chose qui compte -, il faut maintenant analyser deux jugements qui prennent valeur de standards.

18. Ibid., p. 222. Sur la théorie de la Relativité considérée par Meyerson comme une confirmation directe de la « rationalité identificatrice ", citons cette conversation avec Fred Lefèvre parue sous le titre « Une heure avec Émile Meyerson », Les Nouvelles littéraires, 6 nov. 1926, p. 1 : « J'y suis parvenu au point qu'en plaisantant, on a pu dire qu'Einstein avait inventé la théorie de la relativité pour donner une justification actuelle à mes thèses. »

19. Albert Einstein. Philosopher-scientist, éd. Arthur P. ScHILPP, Evanston, IL, Library of living philosophers, 1949.

20. Michel PATY, Einstein philosophe. La physique comme pratique philosophique, Paris, Presses universitaires de France, 1993. 
En citant d'abord, et à nouveau, Paty $^{21}$ :

"Bien qu'important philosophe en son temps, Meyerson est pratiquement oublié aujourd'hui. L'une des raisons de cet effacement réside probablement dans l'importance prise en France par la pensée de Bachelard, laquelle s'est en grande partie constituée par réaction ou polémique contre les thèses de l'auteur d'Identité et réalité. Cette occultation est certainement injuste; si certaines thèses de Meyerson paraissent difficilement défendables aujourd'hui, par ex., l'homogénéité entre pensée commune et pensée scientifique, ses analyses épistémologiques sont souvent pertinentes. C'est ainsi que Bachelard n'a pas saisi, à mon sens, ce que Meyerson entendait par “ déduction " relativiste. Mais j'y reviendrai ailleurs ${ }^{22}$."

Lorsqu'on compulse précisément ce qui devrait être cet «ailleurs » d'une explication contre Bachelard et pour Meyerson, à savoir l'ouvrage Einstein philosophe déjà cité, on ne trouve l'auteur de La Valeur inductive cité, en tout et pour tout, qu'une seule fois, et en des termes qui manifestent les conséquences d'une apparente erreur de lecture, attribuant ainsi à Bachelard une position purement et simplement opposée à celle défendue tout au long de l'ouvrage de 1929 :

« S'il est un sujet de controverses sur les conceptions d'Einstein en physique, et en particulier sur ce que fut sa démarche propre, c'est bien celui du rôle respectif accordé à l'expérience et à la théorie. On lui a attribué tantôt des tendances empiristes, tantôt des inclinations à la spéculation, voire des tendances mêlées et contradictoires qui illustreraient un "opportunisme épistémologique" de bon aloi en recherche; mais ces jugements ont été portés en fonction de thèses à faire valoir, plutôt que de l'analyse de ses idées.

Le thème de l'expérience de Michelson et Morley et de son rôle dans la théorie de la Relativité a suscité, sous ce rapport, des interprétations variées. Selon les vues empiristes dominantes dans le milieu scientifique, et qui soustendent souvent la rédaction des ouvrages de physique, le résultat négatif de

21. Voir Jean Langevin et Michel Paty, "Le séjour d'Einstein en France en 1922 ", in Cahiers Fundamenta Scientiae, 93, 1979.

22. M. PATY, ibid., p. 39-40. Sur l'« indéfendable aujourd'hui " de la thèse de l'homogénéité entre " pensée commune " et « pensée scientifique », l'auteur nous semble se contredire. Ainsi, p. 384 de son Einstein philosophe, op. cit. supra n. 20, il citera (apparemment de manière hagiographique) cette phrase d'Einstein : « La science tout entière, écrit Einstein dans son article de 1936 'Physique et réalité', n'est rien d'autre qu'un raffinement de la pensée de tous les jours »; suit une note : "On trouve aussi cette idée notamment chez Mach; par ex. Enst Mach, Erkenntnis und Irrtum. Skizzen zur Psychologie der Forschung, Leipzig, J. A. Barth, 1905; et chez Meyerson, Identité et réalité, Paris, Alcan, 1908, De l'explication dans les sciences, 2 vol., Paris, Payot, 1921, La Déduction relativiste, Paris, Payot, 1925, Du cheminement de la pensée, 3 vol., Paris, Alcan, 1931. Langevin, proche intellectuellement d'Einstein et de Meyerson, la partageait également. » 
l'expérience, acquis dès 1887 , serait la pierre d'angle de la théorie de la Relativité restreinte, qui se serait édifiée de manière inductive sur cette expérience cruciale. Max Born lui-même, auteur de l'un des meilleurs exposés sur la théorie de la Relativité ${ }^{23}$, fait sienne cette vue lorsqu'il écrit (dans un texte de vulgarisation, il est vrai): "En 1905, Einstein prit [1'échec de l'expérience de Michelson et Morley] comme point de départ de ses réflexions, et érigea en principe le fait que la vitesse de la lumière ne dépend absolument pas des mouvements de l'observateur ${ }^{24}$." Telle est également l'interprétation qu'ont fréquemment donnée les philosophes eux-mêmes ${ }^{25}$. Les adversaires de la théorie de la Relativité, empiristes pour la plupart, soulignaient quant à eux, aux moments forts du débat des années vingt, combien la base expérimentale de la nouvelle théorie (" une simple expérience de second ordre") était ténue en regard de l'“ évidence massive" d'innombrables données expérimentales en faveur de la mécanique classique ${ }^{26}$. »

Nous ne nous attarderons pas ici sur l'exercice d'une critique facile concernant l'incohérence philosophique d'un style et d'une argumentation des plus filandreux : à quoi, à qui renvoie, par exemple, ce « quant à eux » désignant « [les] empiristes pour la plupart »? Sont-ce les mêmes que ceux dominant «dans le milieu scientifique » au point d'y impliquer Born en personne?

Beaucoup plus grave encore, l'alignement inouï de Bachelard sur les traces de Joseph Petzoldt, de Hans Reichenbach ou de... Adolph Grünbaum. Qu'affirme en réalité l'auteur de La Valeur inductive sur cette question précise de l'expérience de Michelson et Morley?

23. Max BorN, Die Relativitätstheorie Einsteins und ihre physikalischen Grundlagen, elementar dargestellt, Berlin, Springer, 1920.

24. M. BoRn, Von der Verantwortung des Naturwissenschaftlers, Munich, Nymphenburger Verlagshandlung, 1965, trad. franç. par L. Jospin, La Responsabilité du savant dans le monde moderne, Paris, Payot, 1967, dans le texte sur «Les limites du monde ».

25. En part., selon les sources de M. Paty et outre G. Bachelard lui-même, Joseph PETzoLdt, "Die Relativitätstheorie im erkenntnistheoretischen Zuzammenhange des relativischen Positivismus behandelt ", Verhandlungen der deutschen physikalischen Gesellschaft, 14, 1912; ID., "Die Relativitätstheorie der Physik ", Zeitschrift für positivistische Philosophie, 2, 1914; Hans Reichenвach, Philosophie der Raum Zeit Lehre, Berlin, De Gruyter, 1928; ID., "The philosophical significance of the theory of relativity ", in op. cit. supra n. 19, p. 289-311; ID., "The philosophical dialectic of the concepts of relativity ", in op. cit. supra n. 19, p. 563-580; Adolph GrúnBaum, «Logical and philosophical foundations of the special theory of relativity ", in Philosophy of science, éd. Arthur Danto et Morgenbesser, New York, Meridian Books, 1960; ID,, " The special theory of relativity as a case study of the importance of the philosophy of science for the history of science ", in Philosophy of science. The Delaware Seminar, éd. B. Baumrin, vol. 2, New York, Interscience, 1963. Au contraire, Henri Bergson, Durée et simultanéité. Á propos de la théorie d'Einstein, Paris, Alcan, 1922, Ernst CASSIRER, Zur Einstein'schen Relativitätstheorie, Berlin, Bruno Cassirer, 1921, É. MeYERSON, op. cit. supra n. 16, ont bien vu que l'approche d'Einstein était différente.

26. M. PatY, "The scientific reception of relativity in France", in The Comparative Reception of Relativity, éd. Thomas GuICK, Dordrecht, Reidel, 1987. 
Renvoyant à l'ouvrage de M. N. R. Campbell, Théorie quantique des spectres. La Relativité ${ }^{27}$, Bachelard affirme sans ambiguité possible ceci :

«M. N. R. Campbell a marqué fortement cette position a priori et formelle des principes de la Relativité. [...] la Relativité cherche quel retentissement les transformations extérieures cinématiques peuvent avoir sur les caractères dynamiques intérieurs des systèmes considérés.

Ce n'est d'ailleurs pas l'expérience qui sera amenée à résoudre ce problème. [...] On ne peut objecter ici l'expérience de Michelson qui serait précise au deuxième ordre de l'approximation, si elle était positive. En effet, dans toute cette expérience, l'observateur terrestre prétend susciter un observateur absolu, lié à l'éther. Cet observateur absolu reste fictif. Au surplus, c'est précisément cet observateur absolu, ou pour parler d'une manière plus expérimentale, c'est le physicien chargé de l'expérience "longitudinale " qui n'observe rien. Il ne voit pas la composition de la vitesse de translation avec la vitesse de la lumière. Le Relativiste ne voit d'ailleurs pas davantage la contraction de Lorentz. Il ne l'expérimente pas. Il l'infère. Il la prend comme une possibilité; il s'aperçoit ensuite qu'elle se coordonne à un système de formes. On est donc bien dans le domaine de l'a priori, non de l'expérience.

Mais une combinaison d'expériences non réalisables peut fort bien conduire, remarque encore Campbell, à des prévisions concernant des expériences possibles. Il suffit pour argumenter que les premières expériences soient concevables. C'est donc à la simple question de la concevabilité des principes qu'il faudra d'abord s'attacher. Puis on postulera que ce qui est concevable est possible. Autrement dit, on se référera à une possibilité a priori, qu'aucune expérience n'instruit, qui n'est gardée que par les limites de l'impossibilité rationnelle. On aura donc, croyons-nous, légèrement élargi la possibilité du réel pour en faire une possibilité de l'idéal. Ce qui nous guidera, ce ne sera pas l'expérience réalisable - c'est-à-dire ce qui est possible expérimentalement - , mais l'expérience concevable, c'est-à-dire ce qui est possible à imaginer et à coordonner à d'autres expériences possibles.

Il y a là, à notre avis, une nuance très faible qui cependant décide de tout, et c'est dans la confusion de ces deux possibles que prennent naissance les différends entre les relativistes et les physiciens de laboratoire. Les physiciens pensent toujours à des expériences réelles, les relativistes à une possibilité d'ordre mathématique. Or, il s'agit d'une expérience possible, mais tout à fait irréalisable. C'est parce qu'elle est irréalisable qu'on peut la définir parfaitement comme une forme, comme un a priori, sans souci des limitations qu'impose une expérience réelle, sans égard pour le caractère plus ou moins approximatif et pour la multiplicité des différentes déterminations. Les principes de la Relativité ont ainsi par un certain côté un caractère véritablement absolu $^{28}$.»

Et quatre pages plus bas, Bachelard poursuit :

27. Trad. Convisy, Paris, Gauthier-Villars, 1924, p. 117.

28. G. BACHELARD, op. cit. supra n. 7, p. 141-144. 
« Finalement, c'est le signe $c$ plutôt que le nombre $3 \times 10^{10}$ centimètres qui compte. Ce signe symbolise moins une quantité qu'une qualité. Toute la connaissance que nous en désirons a trait à son rôle, à ses relations. Au lieu de ses caractères intrinsèques, $c$ 'est surtout ses caractères formels que nous nous efforçons de dégager. Autrement dit, nous sommes moins portés à l'étudier comme un objet que comme une fonction.

D'ailleurs, le côté formel est associé à un caractère apriorique encore plus fortement marqué. L'expérience de Michelson n'est, après tout, que la cause occasionnelle de la découverte d'une "vitesse fondamentale". En effet, en reprenant la construction par l'algèbre, en s'appuyant sur des conditions entièrement a priori concernant les transformations de coordonnées de l'espacetemps, on trouve que l'invariance ne peut être obtenue qu'en introduisant une vitesse qui joue un rôle spécial et que nous appelons pour cela vitesse fondamentale [...] Au surplus, comment une notion expérimentale pourrait-elle interférer avec des notions axiomatiques, sans d'abord être pourvue elle-même d'un caractère axiomatique? Telle est cependant le cas de la vitesse de la lumière qui entre dans la définition a priori de la simultanéité ${ }^{29}$."

Voilà pour la définition de la $c$-fonction ou du $c$-objet, tout à l'opposé d'une quelconque touche empirico-expérimentaliste.

Et puisque les deux textes sont convoqués par Paty pour justifier cet injustifiable, quelles sont les occurrences de cette expérience dans le texte hommage de $1949^{30}$ ? Sur ce topos, désormais traditionalisé en une véritable aberration interprétative, on trouve le même lieu commun chez Pierre Thuillier dans le numéro monographique du mensuel La Recherche consacré à Einstein ${ }^{31}$ :

« Beaucoup de physiciens, d'historiens ou de philosophes accordent en effet à [l'expérience de Michelson-Morley] une énorme importance. D'aptès eux, la science consiste en "généralisations" ou en "inductions" directement fondées sur les faits. La théorie de la relativité était en quelque sorte la réponse " objective " qu'entraînait l'apparition d'un " fait " nouveau.

[...] Ainsi Milikan a fait cette déclaration: "On peut considérer que la théorie de la relativité restreinte a son origine essentiellement dans une généralisation de l'expérience de Michelson. "Einstein, ajoutait-il, est véritablement un scientifique parce qu'il a "rejeté toutes les conceptions a priori concernant la nature de la réalité ". Cette interprétation, à quelques variantes près, revient souvent. Pour le positiviste Joseph Petzoldt, la théorie de la relativité confirmait la faillite de la vieille métaphysique. Seuls comptent les faits : " La théo-

29. Ibid., p. 148-149 et 150 .

30. G. BACHELARD, "The philosophical dialectic of the concepts of relativity", in op. cit. supra n. 19, vol. II, p. 563-580. Version française in G. BACHELARD, L'Engagement rationaliste, préf. de Georges Cangullhem, Paris, Presses universitaires de France, 1972.

31. La Recherche, 96, janv. 1979, p. 20 du « Cas Einstein ». 
rie einsteinienne dépend entièrement du résultat de l'expérience de Michelson et peut en être dérivée ${ }^{32}$." Gaston Bachelard, dont l'épistémologie " dialectique " est pourtant assez éloignée du positivisme, ne tenait pas un langage tellement différent ${ }^{33}$."

Il nous semble évident que l'inducteur de semblables aberrations de lecture n'est autre que l'occurrence du concept d' « induction » en titre. Ce qui a dû autoriser ces auteurs à ne pas dépasser la lecture de son intitulé...

Autre tentative d'exhumation de Meyerson, celle de Catherine Chevalley ${ }^{34}$, relayée par un article de Jean Largeault ${ }^{35}$. Nous citerons d'abord le texte inédit de $\mathrm{C}$. Chevalley, car c'est sur lui que fera ouvertement fonds l'article de Largeault :

«Il est impossible, en présentant un exposé sur la philosophie de Meyerson, d'esquiver la question préalable de l'intérêt d'une telle tentative. Pourquoi consacrer du temps aujourd'hui à lire une œuvre qui a été presque complètement négligée en France depuis la guerre?

À cette question on peut répondre, naturellement, en invoquant d'abord l'intérêt historique. Meyerson est une figure centrale pour l'étude de la réception de la théorie de la Relativité et de la Mécanique quantique. Il appartient au groupe des gens qui jouent un rôle actif dans ce domaine : Langevin, de Broglie, Léon Brunschvicg, André Metz, André George, etc. Il est salué comme le meilleur philosophe des sciences par de Broglie (Introduction à Réel et déterminisme dans la physique quantique, 1933, reprise dans Essais, 1935), et considéré par Einstein comme "un homme qui a su saisir les voies de pensée de la physique moderne et qui a, de plus, profondément pénétré l'histoire de la philosophie et celle des sciences exactes "(Revue philosophique, compte rendu de la Déduction relativiste, 1925). Cette admiration, extrême dans son expres-

32. G. Holton, op. cit. supra n. 10, p. 262.

33. Pierre Thuillier, art. cit. supra n. 31, p. 19-20. Ici, P. Thuillier ne renvoie qu'au texte de 1949 , ignorant délibérément le texte de $1929 .$. 1984.

34. Groupe de travail sur la physique : «Meyerson. La raison et le réel ", séance du 24 mai

35. Jean Largeault, «Émile Meyerson, philosophe oublié », Revue philosophique, 3, 1992 , p. 273-295. Catherine Chevalley nous a confié d'une part, concernant Émile Meyerson, qu'il était un ami intime de son père Claude Chevalley et, d'autre part, que son premier contact avec l'œuvre bachelardienne était passé par la préparation à l'agrégation de philosophie, alors qu'elle était l'étudiante de Michel Serres. La question qui se pose ici concerne à la fois l'image institutionnelle et « officialisée » de Bachelard, bête curieuse des grands concours français, et le double visage du vieil homme urbain à la grande barbe blanche. Guy Petitdemange me rappelait combien toute une génération d'étudiants n'avait connu de lui que l'autre versant de l'ouvre : la face dite « littéraire et poétique ». Quant à l'« autre aspect », celui du difficile travail de philosophie des sciences, de par sa réduction à un pur et simple jeu de formules toutes faites (« la coupure épistémologique », « l'obstacle épistémologique », « le nouvel esprit scientifique $»$ ), facilement intégrées dans les « digests » des manuels de classe Terminale, il aura le plus souvent (au mieux) laissé indifférents nombre d'étudiants, et (au pire) dégoûté une grande majorité d'entre eux. 
sion (même après 1933), est partagée aussi par Koyré, par Lévy-Bruhl, par tout le monde, disons, sauf Bachelard. On peut donc penser qu'en lisant Meyerson, on se trouve devant l'une des formes les plus achevées de la philosophie de la physique en France dans les années 1930, à une époque où la philosophie française avait la réputation d'être la plus active du monde [voir H. Høffding, in Correspondance entre H. Høffding et É. Meyerson, 1939, p. 33: "Il n'y a aucun pays maintenant dans lequel la discussion philosophique soit aussi vive et aussi fertile qu'en France. Les Anglais 'théologisent' trop, et les Allemands sont encore trop entêtés dans leur kantisme "].

[...] Quelle est la signification proprement philosophique de cette œuvre immense? Meyerson, en effet, a voulu faire une æuvre de philosophe. Il a voulu, en remontant des sciences vers les questions les plus anciennes et les plus fondamentales de la théorie de la connaissance, et en se servant de l'histoire des sciences et de l'analyse de la physique moderne, parvenir à un éclaircissement du problème des rapports de la raison et du réel.

[...] Ce travail conduit à faire une hypothèse au sujet de ]'oubli dans lequel est tombée l'œuvre de Meyerson : celle que cet oubli tient à la dépendance de cette œuvre à l'égard d'un contexte philosophique que l'on connait aujourd'hui très mal. Ce contexte est celui de la réflexion sur la Relativité et la Mécanique quantique en tant que cette réflexion est déterminée par la nécessité d'avoir à se situer par rapport à la critique bergsonienne de Kant. C'est donc le contexte bergsonien, mais en tant que le statut du kantisme y est constamment discuté. Ce qu'on a oublié, c'est l'appréciation du niveau philosophique auquel se situaient les débats. L'épistémologie bachelardienne a donné, ensuite, l'impression que l'on pouvait réfléchir directement sur la science en-dehors de l'histoire philosophique ${ }^{36}$, mais elle l'a fait en réglant violemment les comptes avec Meyerson, et, à travers lui, avec Bergson. Par conséquent, en lisant Meyerson, on voit émerger tout ce contexte du bergsonisme, avec, en arrièreplan, Renouvier, Boutroux et Ravaisson [...].

Meyerson a fait son éducation philosophique avec Renouvier [Cf. Correspondance, cit. p. 84] et se réclame de Boutroux, Bergson, Poincaré et Duhem [Identité et réalité, p. xvm]. [...] Bref, il me semble que l'cuure de Meyerson a péri de la même mort que celle de la philosophie française idéaliste de cette période! »

\section{Une dernière citation de C. Chevalley qui n'est pas la moins étonnante :}

" Il faut donc dire, une fois pour toutes, que les livres de Meyerson répondent en fait, à presque toutes les exigences énumérées par Bachelard. Ils sont précis,

36. Sur cette pseudo-sortie bachelardienne " hors de l'histoire ", voir l'affirmation opposée de la tradition italienne pourtant historiciste, par ex., Carlo MANZONI, L'Epistemologia di Émile Meyerson, Rome, Ed. dell'Ateneo, 1971. C'est très précisément ce qui a pu être défini comme une «épistémologie historique " qui constitue pour C. MANzonI, ibid., p. 8, le seul point de rapprochement possible de Meyerson avec Brunschvicg et Bachelard : «Malgré leur diversité, on pourra constater comment un motif identique conditionne ces trois auteurs dans leur pratique de l'" histoire des sciences", et comment, en réalité, l'acception d' "épistémologie historique" peut leur être attribuée, avec toutes les différences évidentes et radicales [qui les séparent]. " 
rigoureux, écrits en "langue naturelle", extraordinairement informés des développements les plus récents de la physique et de la philosophie mathématique, extraordinairement érudits, également en matière d'histoire des sciences. Ce qui est déconcertant aujourd'hui, du moins pour les philosophes formés après-guerre - c'est qu'il a tendance à un certain éclectisme dans ses citations, et que sa connaissance de l'histoire de la métaphysique est parfois, comme il l'a lui-même reconnu, un peu trébuchante. Mais ce défaut est beaucoup moins sensible quand il discute Poincaré, Helmoltz, Weyl ou Eddington, et à bien des égards Meyerson apparaît comme l'un des premiers "épistémologues " au sens actuel du terme. Il s'est d'ailleurs présenté comme un "philosophe de l'intellect" dans la voie ouverte par Kant, et non comme un philosophe de la nature."

Sur la diagnose post mortem de C. Chevalley concernant cette double mort de Meyerson et de "l'école idéaliste française de la période », nous lui concéderons certes que Bachelard aura bel et bien toujours cherché à annuler toute cette littérature que Desanti qualifiait en 1973 de « contreépistémologie ${ }^{37}$ ».

C'est donc en 1992, que Largeault remettra sur le métier cette approche dans la Revue philosophique:

« [...] les contributions de Meyerson aux discussions sur les nouvelles théories physiques furent appréciées par Einstein et de Broglie. Cette grande estime, que partagent Koyré, Lévy-Bruhl, est unanime, à l'exception de Bachelard, qui a une conception opposée à celle de Meyerson sur un point qu'on aurait tort de croire de mince importance. [...] primo, selon Bachelard, il y a une "coupure" (une "démarcation "), là où Meyerson n'en voit aucune [...] Secundo, selon Meyerson, un réalisme est inhérent à la recherche et aux théories scientifiques prolongeant celui du sens commun. Dans la perspective de Bachelard, le réalisme est contraire à l'esprit scientifique.

L'épistémologie de Bachelard a oblitéré celle de Meyerson, qui comptait pour un ensemble de réflexions le plus proche de la vérité sur la physique, du temps que la philosophie française était réputée "la plus active du monde" (H. Høffding). »

Après avoir examiné ces oublis et mésinterprétations qui apparaissent avant tout comme l'effet de non-lectures de Bachelard, on peut en venir à l'enjeu épistémologique comme tel de cette opposition. Si la question touche aux «philosophes lecteurs d'Einstein », il est possible d'affirmer tout simplement que l'interprétation, et plus encore la démarche de Meyerson et de Bachelard sont manifestement antinomiques ${ }^{38}$. Or, les développe-

37. Jean-Toussaint DeSANTI, «Sur Bachelard», Cours du $1^{\text {er }}$ semestre 1973-1974, École normale supérieure de Saint-Cloud, dactylogr.

38. Sur cette opposition évidente, plus particulièrement centrée ici autour de La Valeur inductive de la relativité et de La Déduction relativiste, voir, pour une première approche : Dominique Lecourt, L'Épistémologie historique de Gaston Bachelard, Paris, Vrin, 1969, et 
ments récents de la physique semblent avoir brouillé les cartes dont ils disposaient, et conduire - ce qui explique ainsi un certain retour fantasmatique à Meyerson - à une inversion de leurs interprétations. Pour le dire déjà par anticipation, Meyerson nous présente en 1925, dans La Déduction relativiste, un Einstein physicien classique, Bachelard insistant quant à lui, à peine quatre ans plus tard, sur la totale nouveauté de la Physique relativiste.

Au vue des raisons avancées à l'époque, il semblerait que ce soit Bachelard qui ait raison. Aujourd'hui pourtant s'impose de plus en plus l'idée qu'Einstein est très certainement le dernier des grands physiciens classiques. On retrouverait alors, à ce qu'il semble, la leçon de Meyerson.

Où donc se noue le chiasme ? Et laquelle de ces deux déhiscences épistémologiques vaut-elle encore aujourd'hui?

En bref, la question fondamentale qui travaille la vision meyersonienne de la Relativité part du constat que « la tendance idéaliste et les convictions réalistes [y] coexistent côte à côte ". S'il est bien vrai que c'est la recherche des invariants qui constitue l'essentiel de l'activité du physicien relativiste, Meyerson en conclut que "le réel de la physique relativiste est un absolu ontologique, un véritable être en soi ". C'est là sa conviction réaliste profonde. Ce qui exprime son souci d'arracher la physique d'Einstein à l'interprétation positiviste qui en fut donnée. Mais reconnaissant la dimension réaliste de la Relativité, Meyerson ne méconnaît pas pour autant le rôle des mathématiques. La Physique relativiste c'est aussi un mathématisme ou, comme il le dit souvent, un pangéométrisme. On retrouve ici toutes les caractéristiques mathématiques de principe sur lesquelles insiste Brunschvicg : le rôle du calcul, l'importance de la déduction mathématique, et l'idée que la physique d'Einstein est une construction mathématique. Dans cette construction, Meyerson souligne l'importance de la géométrie, considérant que la notion fondatrice est ici celle d'Espace. C'est que le relativisme vient s'arc-bouter sur une rationalité à la fois identificatrice et spatialisante.

Ce qui est au fond de toute sa réflexion épistémologique, c'est cette thèse selon laquelle la rationalité est fonction d'identification (au sens fort de l'identité), «schéma le plus général de tous nos raisonnements sans exception ${ }^{39} »$. Le principe d'identité constitue par conséquent «le cadre éternel de notre esprit », "la véritable essence de la logique, le vrai moule

\footnotetext{
Georges Mourélos, L'Épistémologie positive et la critique meyersonienne, Paris, Presses universitaires de France, 1962.

39. É. Meyerson, De l'explication dans les sciences, Paris, Payot, 1921 (2 vol.), $1927^{2}$ (1 vol.) ici citée, p. 620. La collection "Corpus des cuvres de philosophie en langue française ", Paris, Fayard, 1995, en a reproduit l'édition, revue par Bernadette BENSAUDE-VINCENT, malheureusement inutilisable par non-concordance des paginations.
} 
où l'homme coule sa pensée ${ }^{40}$ ". De plus, ce principe «constitue la plus vaste des hypothèses que nous puissions formuler, puisqu'il s'applique à la totalité du monde sensible ${ }^{41} »$. Plus fondamental encore, «identification ici signifiera donc non seulement l'acte par lequel nous reconnaissons l'identique là où il existe, mais encore l'acte par lequel nous ramenons à l'identique ce qui nous a paru, tout d'abord n'être pas tel ${ }^{42} »$. Cette activité identificatrice de la raison n'est pas cependant une pure activité tautologique, mais une sorte de procédé synthétique impliquant un progrès de la pensée : «Il ne formule pas l'identité déjà préexistante dans notre esprit, déjà reconnue, ce qui serait oiseux, il la fait reconnaître, il l'introduit là où nous ne reconnaissions pas son existence ${ }^{43}$."

Par ailleurs, la question de l'identité est étroitement liée à celle de la causalité, car « le principe de causalité n'est que le principe d'identité appliqué à l'existence des objets dans le temps ${ }^{44}$ ". La science est explication causale, et l'explication causale est procès d'identification en ce sens précis qu' « en recherchant l'explication du phénomène, ce que le physicien poursuit en réalité, c'est la démonstration que l'état conséquent ne diffère point du précédent, mais peut au contraire être considéré comme lui étant identique $^{45} »$. Or, pour que cela puisse se réaliser, il est nécessaire d'éliminer le temps comme facteur du changement et du devenir, et de réduire toute la réalité à sa seule dimension spatiale. Meyerson déclare explicitement : " Le postulat causal consiste à nier, à éliminer l'influence du temps. Il ne nous reste que l'espace... L'explication la plus parfaite d'un changement ne peut consister qu'en sa réduction à une fonction spatiale ${ }^{46}$. " Dès lors, le " géométrisme » meyersonien vient s'inscrire au cour de cette double contrainte d'une rationalité identificatrice et spatialisante. C'est pourquoi, si le « relativisme » ne fait que porter à ses extrêmes conséquences un mouvement naturel de la science, il privilégiera la forme d'explication spatiale géométrique plutôt que la forme algébrique ${ }^{47}$ : «C'est que dans le relativisme

40. É. MeYerson, Identité et réalité, Paris, Alcan, 1908, $1912^{2}$ (éd. rev. et augm.), ici $1951^{5}$, p. 322 et 337.

41. Ibid., p. 459.

42. É. MEYERSON, op. cit. supra n. 39, p. 451.

43. Ibid., p. 145.

44. É. Meyerson, op. cit. supra n. 40 , p. 38.

45. Ibid., p. 49.

46. É. MeYerSOn, op. cit. supra n. 39, p. 159.

47. Sur ce point, voir, à nouveau, la position radicalement antagoniste de G. BACHELARD, in op. cit. supra n. 7, p. 80, définissant fort exactement l'essence de la méthode tensorielle en Relativité généralisée par induction algébrique : « La simple transformation algébrique va nourrir les formes aux dépens les unes des autres et équilibrer leur valeur réalistique [...], affermir les cadres de la possibilité pure. [...] Réalité et possibilité vont se trouver subsumées sous une totalité d'un ordre algébrique particulièrement homogène. " Voir également, in ibid., p. 47,76 et 78 , ainsi que notre commentaire technique qui constituera le second volet de cet article. 
considéré en son aboutissement complet, tout est géométrie et n'est que cela $^{48}$.» Le géométrique, comparé au pur algébrique, recèle encore « un élément qui n'est point d'essence purement rationnelle, un élément de qua$\operatorname{lité}^{49} \gg$. C'est bel et bien là le véritable prix, et non la limite, de l'explication relativiste "qui fait que le spatial nous apparaît comme doué d'une certaine réalité, et dès lors comme apte à nous représenter le réel, à l'encontre de ce qui a lieu pour le purement algébrique ${ }^{50} \gg$. Ce serait là le privilège exclusif de l'explication géométrique :

« $[\ldots]$ ce que l'on met à la place du physique doit tout de même pouvoir être conçu, à certains égards, comme un réel, cela doit, jusqu'à un certain point, pouvoir s'imaginer. Alors que, devant de pures fonctions algébriques, notre imagination s'arrêterait découragée ${ }^{51}$.»

C'est précisément ce point qu'Einstein, dans sa recension de 1927, lui refusera résolument :

« Ici [...] il y a une confusion à éviter dans l'interprétation de certaines affirmations de M. Meyerson, et notamment celle-ci : “La relativité ramène la physique à la géométrie." Il est très exact qu'avec cette théorie la géométrie (métrique), regardée comme distincte des disciplines jusqu'alors classées sous la rubrique "physique", a perdu son existence indépendante. [...] Cela ne suffit cependant point à justifier l'application du nom de "géométrie " à toute science où [la forme mathématique] joue un rôle [...]. Ainsi, je suis d'avis que le terme de "géométrique" employé dans cet ordre d'idées est entièrement vide de sens ${ }^{52}$.»

Enfin, origine et horizon de tout ce dispositif meyersonien, la condition de la science se trouve dans l'existence d'un Réel ${ }^{53}$ qui, d'abord, n'apparaît pas comme intelligible et rationnel puisqu'il est qualitatif, diversifié, qu'en lui des discontinuités et des irréversibilités s'expriment. Ce Réel est d'abord opaque à la pensée. Tout l'effort de la science revient à essayer de le rendre intelligible. Dans cet effort, la mathématique joue un rôle déter-

48. É. Meyerson, op. cit. supra n. 16, p. 120.

49. Ibid., p. 40.

50. Ibid.

51. Ibid., p. 223. Voir ce que G. BACHELARD, in op. cit. supra n. 7 , p. 71 et 76 , oppose ici : « On a amené l'algèbre à coopérer avec le réel, par la propre impulsion du calcul, sans jamais poser et chercher l'instruction par le réel comme primordiale ", ainsi que dans ce qu'il qualifie de « syllogisme de la confiance ».

52. Albert EINSTEIN, compte rendu de La Déduction relativiste d'É. Meyerson, Revue philosophique, vol. CV, 1924, repr. in op. cit. supra n. 15, vol. V, « Science, éthique, philosophie », p. 91-92.

53. Voir, par contre, et entre mille, G. BACHELARD, op. cit. supra n. 7, p. 243 : «C'est à tort qu'on veut voir dans le réel la raison déterminante de l'objectivité alors qu'on ne peut jamais apporter que la preuve d'une objectivation correcte. » 
minant puisqu'elle quantifie le donné qualitatif. Mais ce qu'il faut surtout exorciser, c'est la diversité, le pur éparpillement du Réel. Il faut retrouver la permanence dans le devenir, l'unité sous-jacente à la pluralité, l'identité dans la réalité multiple. C'est ce qui apparaît déjà au niveau de la perception commune, et la Science ne fait rien d'autre que de poursuivre ce travail d'unification. On voit à nouveau, sans y insister ici, tout ce qui sépare et oppose radicalement Meyerson de Bachelard.

Mais s'ajoute encore à ce dispositif métaphysique meyersonien, une autre démarche, qui inscrit dès l'abord le conflit des titres, celle de la déduction par laquelle nous expliquons. C'est ici l'essentiel de la thèse de Meyerson : expliquer, c'est déduire.

Selon Meyerson, «comprendre, expliquer veut dire déduire». «Une déduction embrassant un certain nombre de faits s'appelle une théorie. » Dès lors, la « déduction relativiste » n'est rien d'autre que la reprise de cette démarche qui animait déjà la physique pré-einsteinienne, parce qu'elle est la démarche de la Science même. Les relativistes, affirme Meyerson, reprennent à leur compte le programme éternel de la science de toujours, c'est-à-dire en fait, « qu'ils ne font, dans cet ordre d'idées, que pousser - fort loin il est vrai - un mouvement qui est celui de la science : c'est tout simplement l'éternelle tentative visant à expliquer le devenir, le changement dans le temps, par la négation de ce changement ${ }^{54}$ ".

On n'a jamais compris le changement qu'en essayant de le nier. C'est toujours la même Raison. Sur ce point, Meyerson s'oppose à Hegel : « La Raison a été identique à elle-même, à toutes les époques, et en toutes circonstances. " L'idée d'une évolution de la raison est scandaleuse. Ce qui s'est transformé, c'est ce qu'il appelle « la Raison géométrique », c'est-àdire l'espace qui fait partie de la raison comme un outil fait partie du travail qu'il exécute.

Mais si les instruments de la raison se transforment, ses fondements restent immuables. La raison est par essence absolue; elle n'admet point quoi que ce soit dans le réel qui lui échappe. Tout doit lui être soumis, tout doit apparaître comme rationnel: ainsi, la «Raison relativiste » est l'expression actuelle de cette tentative de rationalisation du réel, et elle n'a donc rien de révolutionnaire ${ }^{55}$. D'où son caractère classique du point de vue des démarches de la pensée : c'est la même raison que celle dont usait Descartes, et à laquelle se référait Newton, que nous trouvons à l'œuvre dans la Physique relativiste d'Einstein. Rien de nouveau sous le soleil de la Raison.

54. É. MeYerson, op. cit. supra n. 16, p. 108.

55. Ibid., p. 300 : «Les relativistes se sont bien gardés de proclamer l'avènement d'une raison nouvelle, ils ont au contraire tout fait pour maintenir la croyance qu'ils raisonnaient comme on avait toujours raisonné. » 
Bachelard insiste quant à lui, et dès les premières lignes de son texte, sur la nouveauté radicale des doctrines relativistes. Cette nouveauté est telle qu'il est difficile de trouver, dans l'histoire des sciences ou de la philosophie, des antécédents à la physique d'Einstein. La Relativité est une de ces « sciences sans aieux » dont il parlera plus tard, instaurant une coupure et s'inscrivant dans une vision de discontinuité : "S'il est une doctrine que les antécédents historiques n'expliquent pas, c'est celle de la Relativité. "

D'où vient cette nouveauté?

Si pour Bachelard (comme pour Meyerson) le relativiste est bien un réaliste $^{56}$, il donne à ce réalisme une tout autre allure. Il parle de " foi réaliste » du relativiste et de son « ardeur militante ». Pour lui, le physicien ne se contente pas de découvrir le réel, il le construit :

« Il n'est évidemment question que d'une référence d'ordre purement mathématique, au centre d'une construction d'ordre uniquement mathématique. Il ne s'agit pas de trouver et de décrire une réalité qui se désignerait à nous par des caractères mathématiques particuliers. C'est au contraire une tâche constructive qui s'impose au théoricien.

[...] [O]n voit la Réalité relativiste apparaître à l'extrême pointe d'une construction; elle est clairement solidaire d'une méthode de construction ${ }^{57}$. »

Si en relativité « le réel se démontre, il ne se montre pas ${ }^{58}$ », et c'est bien là qu'il faut placer la valeur inductive de la Relativité, sa force d'inférence, c'est-à-dire de construction. «Induction » est ici à entendre en un sens tout à fait spécifique, en ce sens bachelardien qui est mis en œuvre dès sa thèse principale du doctorat ès lettres soutenue en Sorbonne le 23 mai 1927 devant Abel Rey et Léon Brunschvicg ${ }^{59}$. La Relativité n'est pas tant une déduction explicative, qu'une

« [...] construction. [...] [L]a pensée qui l'anime se place résolument devant une tâche constructive où elle cherche les compléments, les adjonctions [...]. Autrement dit, la nouveauté relativiste n'est pas d'essence statique; ce ne sont

56. Contre les conventionalistes, c'est-à-dire « contre l'indifférence excessive de ces théoriciens qui ne voient dans un système scientifique qu'un moyen plus ou moins commode de résumer l'expérience ».

57. G. BACHELARD, op. cit. supra n. 7, p. 157 et 125. Le constructivisme bachelardien est partout émergent et en permanence opératoire dans cet ouvrage. Il sera définitivement récurrent dans l'œuvre.

58. Ibid.

59. G. BACHELARd, Essai sur la connaissance approchée, Paris, Vrin, 1927. Cette thèse, qui « a été couverte de fleurs à la soutenance » (selon G. RaBEAU, voir supra n. 13; voir, également, Henri GouHIER, dans les Nouvelles littéraires du 10 mars 1928, p. 10), définit à nouveaux frais cette notion opératoire, voir ibid, chap. vill, p. 127; “ On croit souvent que l'induction est, pour la connaissance, uniquement un principe d'acquisition. En réalité, elle n'a pas seulement une valeur pour acquérir, mais pour conserver. Conceptualisation et induction représentent la même opération. " 
pas les choses qui viennent nous surprendre, mais c'est l'esprit qui construit sa propre surprise et se prend au jeu de ses questions. La Relativité, c'est plus qu'un renouvellement définitif dans la façon de penser le phénomène physique, c'est une méthode de découverte progressive ${ }^{60}$. "

Bref, la valeur essentielle de la Relativité tient donc dans une multiplication et un enrichissement prémédités de considérations théoriques, et même spécifiquement mathématiques, qui ne s'orientent vers l'expérience que tardivement, et par surcroît :

«Tout le long de la construction relativiste, nous avons vu ainsi se déposer des réalités tardivement définies, qui se présentaient comme l'achèvement d'une pensée. La direction de notre effort vers le réel est d'une netteté inflexible. C'est une conquête, non une trouvaille. Notre pensée va au réel, elle n'en part pas $^{61}$."

«Induction » signifie donc ici création. Le réel n'est pas d'abord posé, comme il l'était chez Meyerson.

" C'est au point que nous croyons pouvoir dire en vivant sur le plan de la pensée scientifique renouvelée par l'hyper-criticisme relativiste que l'essence est une fonction de la relation [...]. Au commencement est la relation; tout réalisme n'est qu'un mode d'expression de cette relation; on ne peut pas penser en deux fois le monde des objets : d'abord comme relatifs entre eux, ensuite comme existant chacun pour soi [...]. [L]a relation affecte l'être, mieux, elle ne fait qu'un avec l'être ${ }^{62}$.»

Le relativiste n'est pas un découvreur ou un explorateur de l'être, recherchant l'absolu dans la forêt enchevêtrée des phénomènes ou des observations sensibles immédiates. C'est bien plutôt un inventeur, un constructeur, on pourrait dire même un créateur de ce « réel tardif de la Science ». Si cette création est bien l'œuvre des mathématiques, celles-ci auront alors une fonction toute différente de celle reconnue par Meyerson. La déduction mathématique meyersonienne nous fait rejoindre un réel déjà là, donné dans son irrationalité originaire. Pour Bachelard, il ne s'agit là que d'une démarche explicative, c'est-à-dire seconde. « Avant d'expliquer, il faut construire ». La Physique relativiste étant une construction et non une explication, les mathématiques y ont un rôle beaucoup plus décisif et déterminant que chez Meyerson; devenant dès lors créatrices de l'expérience,

60. G. BACHELARD, op. cit. supra n. 7, p. 6 (c'est la page inaugurale de l'ouvrage).

61. Ibid., p. 241.

62. Ibid., p. 208,210 et 211 . Sur tout ce noyau thématique, je renvoie aux concepts affines engagés (et sous-tendus) par le travail de Laurent NotTale, La Relativité dans tous ses états, Paris, Hachette, 1999 (cf. infra p. 165-174). 
elles opèrent ainsi un déplacement du réalisme. "La Relativité crée son expérience » : voilà l'idée centrale, le sens inhabituel donné à l'induction valorisante.

Si le réel de la Relativité est un réel créé, à partir de quoi pourrait-on créer du réel, sinon à partir du possible? Il faut donc déborder les limites étroites de la réalité telle que nous la donne l'expérience sensible. Il faut ajouter quelque chose au réel immédiat. Et c'est bien ce que fait la Relativité par la considération des systèmes de référence différents du nôtre. « $\mathrm{La}$ pensée qui anime la Relativité procède par adjonctions successives. » Rien qui ressemble ici à la logique déductive, laquelle se contente d'expliciter les virtualités contenues dans les prémisses, et considère la synthèse comme une simple vérification de l'analyse ${ }^{63}$. Il faut généraliser, déborder l'immédiateté sensible, entourer le réel d'une frange de possibles,

63. Sur la centralité de la notion de «synthèse", sa solidarité catégoriale avec le «constructivisme", on peut renvoyer à Paul Langevin. Dans sa Conclusion générale de la Deuxième Semaine de synthèse consacrée à La Relativité, Paris, Hermann (Actualités scientifiques et industrielles, vol. XLV), 1932, p. 3, 4 et 11, il déclare : « Après les quatre belles conférences qui vous ont retracé les différentes phases du développement de la théorie de la Relativité [Edmond Bauer, Francis Perrin, Louis de Broglie et Georges Darmois], il n'est peut-être pas inutile d'insister sur certains points particuliers, intéressants au point de vue de la Synthèse, et de souligner l'importance de la théorie relativiste non seulement pour la physique elle-même, mais aussi pour la philosophie, pour sa liaison avec les autres activités intellectuelles et finalement pour la théorie de la connaissance, c'est-à-dire la réflexion de l'esprit sur sa propre activité. [...] Le succès de la théorie de la relativité, comme moyen de représentation cohérente du monde, constitue en quelque sorte sa justification pragmatique. Son intérêt philosophique réside dans la clarté qu'elle projette sur les notions fondamentales et sur tout le mécanisme évolutif de la pensée humaine [...]. La puissance de synthèse de la théorie de la relativité se manifeste dans tous les domaines. »

Enfin, six ans plus tard, au célèbre congrès de Varsovie de 1938 réunissant Bauer, Bohr, Brillouin, de Broglie, Eddington, Fowler, Gamow, Klein, Kramers, von Neumann, Wigner et bien d'autres, P. LANGEVIN - dans son article « The positivistic and realistic trends in the philosophy of physics ", in New Theories of physics. Reports and discussions (Warsaw Conference, League of Nations). International Institute of Intellectual Co-operation, Paris, Scientific Collection, 1939, p. 222 et 227 - insiste encore sur cette puissance de synthèse constructiviste de la Relativité : "I wish to show that the critical activity which is more static, corresponds to the positivistic attitude, and that contrariwise, constructive activity, the creation of new ideas, corresponds rather to the realistic attitude of the physicist [...]. This constructive aspect, that concerns rather the manner in which ideas are transformed and renewed, which tends to develop and affirm more and more the organic unity of our representation, constitutes for me the theoretical construction such as we see developping before our eyes and such as physicists have created, by detaching more and more the invariant elements, that is to say the elements which are independent of the conditions of experiment or observation [...]. 》 Notons également la convergence stricte du président de ces sessions, C. Bialobrzeski, dans ses remarques finales, in ibid., p. 240 : «The process of construction is thus essentialy synthetic; starting from simple, abstract ideas it rises, by combining them, to more and more complex ideas which become progressively better and better adapted for reproducing, in thought, Nature's realities with their varied attributes. 》

Enfin, sur l'autre versant de la Physique contemporaine, n'oublions pas comment André GEORGE, dans son «Esquisse pour un portrait », in Louis de Broglie physicien et penseur, Paris, Albin Michel, 1953, p. 451, qualifiera le fondateur de la Mécanique ondulatoire, Louis de Broglie : « [l]e grand constructeur. » 
« prendre prétexte du réel livré par l'expérience et [...] l'entourer d'un cortège de possibilités ${ }^{64} \gg$. En somme, possibiliser le réel au lieu de réaliser le possible.

Or, c'est précisément ce que fait le calcul tensoriel qui est au fondement mathématique de la Relativité dite générale. On pourra toujours chercher un chapitre, un paragraphe même, qui serait consacré au calcul tensoriel dans La Déduction relativiste. Pas une fois, me semble-t-il, n'apparaît le terme même de tenseur. Bachelard quant à lui y consacre tout son chapitre II qu'il intitule précisément : «L'induction mathématique dans les doctrines de la Relativité. »C'est sans doute le chapitre qui fait pivot, là où Bachelard y est le plus actuel. Généralisant le calcul vectoriel, le calcul tensoriel poursuit la plus grande richesse possible, la plus grande diversité des variables :

« Le calcul tensoriel nous paraît donc, en son essence, éminemment apte à donner les cadres d'une généralisation. Qu'on lui livre une des variables du problème, il saura lui associer toutes les autres [...] Génie de la généralité [...] il généralise en sensibilisant toutes les variables ${ }^{65}$. »

Sur l'actualité radicale de cette approche pour une réflexion sur la Relativité générale, nous reviendrons en détail dans le second volet de cet $\operatorname{article~}^{66}$.

Pour clore ici la fausse question de la nécessité d'une pseudo-renaissance meyersonienne, on peut dire que, pour Bachelard, ce qu'impose la physique d'Einstein, c'est la nécessité de rapporter les résultats de la mesure aux conditions de la mesure, c'est-à-dire en fait, d'ériger l'invention en méthode. Ce qui exclut tout intuitionnisme de l'immédiat. Les notions les plus évidentes doivent aussi être construites et démontrées. Ainsi, pour l'idée familière de simultanéité : un principe, la constance de la vitesse de la lumière, entre dans sa définition. Tout est construit à partir de principes formels. L'expérience immédiate n'est prise en compte qu'après avoir subi ce travail de formalisation et d'axiomatisation qui l'élève au rang de principe. Un exemple - qui vient s'inscrire absolument en faux contre l'affirmation réductrice de Paty dans son Einstein philosophe: la constance de la vitesse de la lumière n'est pas tant un fait d'observation (résultat négatif des expériences de Fizeau et de Michelson) qu'une règle de construction :

«En effet, dans la Relativité, la vitesse de la lumière n'apparaît plus comme une réalité trouvée dans une expérience, mais plutôt comme une réalité affir-

64. G. BACHELARD, op. cit. supra n. 7, p. 80.

65. Ibid., p. 63 et 72 .

66. Il fera l'objet d'une publication ultérieure. 
mée dans une règle [...]. La réalité apparaît là comme un exemple de construction. Le principe de la construction est intime, il ne vient pas du dehors [...].

Au surplus, comment une notion expérimentale pourrait-elle interférer avec des notions axiomatiques, sans d'abord être pourvue elle-même d'un caractère axiomatique? Tel est cependant le cas de la vitesse de la lumière qui entre dans la définition a priori de la simultanéité [...]. Prises dans cet état de corrélation indissoluble, la vitesse de la lumière et la simultanéité apparaissent donc bien comme deux pures notions, comme deux entités définies a priori et qui ne recevront que par la suite, tant bien que mal, un sens dans l'expérience ${ }^{67}$.»

Si l'on rapproche ces deux lectures ou interprétations de la Relativité, on peut donc dire sommairement qu'en 1925, Meyerson nous présente un Einstein classique, répétant une démarche de pensée qui fut celle de ses prédécesseurs, et qu'en 1929, Bachelard nous propose un Einstein novateur, révolutionnaire par sa considération des systèmes possibles. Pourquoi ? Parce que Bachelard lecteur d'Einstein, et à la différence de Meyerson, n'a pas (et n'aura jamais) une théorie de la connaissance «sous la main » ou dans sa manche. Il ne projette pas un système de pensée sur la physique d'Einstein. Sa philosophie (le système), il le(s) découvre dans cette physique même. Il faut noter à ce propos l'emploi fréquent de l'expression « le système d'Einstein ». C'est que les thèses caractéristiques de l'épistémologie bachelardienne («Connaissance approchée », « polyphilosophisme de la Science », « Rationalisme appliqué ») ne font jamais qu'expliciter les caractéristiques des théories scientifiques analysées par Bachelard. Quant à ses thèses philosophiques, qui pourraient sembler précéder l'analyse épistémologique comme telle (le temps conçu comme suite atomistique d'instants; l'omniprésence du sujet rationnel; la valeur créatrice des mathématiques...), elles s'adossent toujours aux résultats d'analyse sécrétés par le système relativiste lui-même, et elles ne s(er)ont jamais cohérées en un système exogène et préétabli.

La lecture de Meyerson présente quant à elle tous les défauts de la systématique philosophique préjugeante et inchoative ${ }^{68}$. Et c'est cette opposition Meyerson-Bachelard qui dominera toute l'épistémologie française jusqu'à nos jours. Si on peut l'entrevoir ici dans le cas d'un objet précis (la Relativité), plus profondément encore cette opposition apparaît comme celle de deux approches du texte scientifique dont l'une débouche, avec Meyerson,

67. G. Bachelard, op. cit. supra n. 7, p. 145, 150 et 151.

68. ID., L'Activité rationaliste dans la physique contemporaine, Paris, Presses universitaires de France, 1951 , ici $1965^{2}$, p. 12 : « La spécialisation scientifique détermine un attachement de la pensée subjective à une tâche, non pas toujours la même, mais qui veut toujours se renouveler. Cet attachement est la condition d'un vigoureux engagement d'un esprit dans un domaine de recherche. Faute de comprendre cette dialectique de l'attachement et de l'engagement on méconnaît les vertus rénovatrices de la recherche scientifique spécialisée. La culture générale telle que la prônent les philosophes reste souvent une culture inchoative. ” 
sur le style de ce qu'on devrait qualifier de " contre-épistémologie » (ou de «pseudo-histoire" des sciences), abordant l'écriture de la science tout armé d'un "système philosophique a priori », et l'autre, avec Bachelard, sur un style d'épistémologie philosophique (nous devrions même parler d'épistémologie poiétique - c'est-à-dire finalement, de "philosophie tout court »).

Pour ceux qui, cédant à la mode du consensus, prétendent à un possible œcuménisme des deux auteurs (sur le modèle d'un Bachelard soi-disant et finalement bergsonien), je leur jette en pâture deux jugements (entre cents) portés par Bachelard sur Meyerson : «Tel philosophe qui écrit à soixante ans, défend encore la thèse qu'il soutint à trente ans. La carrière entière, chez certains philosophes d'aujourd'hui, est ainsi une "soutenance continuée " ${ }^{69}$. » Et cinq ans plus tôt, dans un texte de conférence inédit : " [...] le philosophe Meyerson qui est, comme je le dis quelquefois d'une manière un peu cruelle, le plus grand des épistémologues du XIx ${ }^{e}$ siècle $^{70}$."

« INDUCTIVTTÉ 》 ET DISPOSITIF D'INDUCTION.

LES ENJEUX DE LA MÉTAPHORE ÉLECTROMAGNÉTIOUE

« Au début de l'ère relativiste, pour prouver la solidité des nouvelles doctrines, Painlevé dit aux ignorants, en parlant des cinquante mathématiciens réunis autour d'Einstein : "Regardez, on les voit se comprendre".,

Gaston BACHELARD, Lautréamont, 1940, p. 119.

Je n'aborderai pas ici un élément important du dispositif inductif que reprendra pourtant le projet bachelardien dans son économie générale : une séquence historico-textuelle que je nommerais volontiers « De Whewell aux réseaux dérivés des grands Inducteurs " (par exemple, William Whewell ${ }^{71}$, Alfred North Whitehead et le concept affine d'« abstraction extensive ", anticipé par Charlie Dunbar Broad dès $1923^{72}$, Ferdinand Gonseth,

69. G. BACHELARD, Le Rationalisme appliqué, Paris, Presses universitaires de France, 1949, p. 43.

70. ID., Conférence du lundi 22 mai 1944, in Mésons et théorie des forces nucléaires. "Le Méson. Aspects théoriques et expérimentaux ". Réunion d'études et de mise au point, Éd. de la Revue d'optique théorique et instrumentale, 1945 (fonds Jean-Louis Destouches). C'est à la générosité de $\mathbf{M}^{\text {mec }}$ Paulette Février que nous devons l'accès à ce texte.

71. Je dois à mon ami Bernard Besnier d'avoir attiré mon attention sur la thèse de Robert Blanché. Voir Robert Blanché, Le Rationalisme de Whewell, Paris, Alcan, 1935.

72. Charlie Dunbar Broad, Scientific Thought, Londres, Routledge \& Keagan Paul, 1923, 1952. Helge S. KraGH, Dirac. A scientific biography, Cambridge, Cambridge University Press, 1990, ici $1991^{2}$, p. $6:$ : In 1920-1921, together with some of his fellow engineering students, P.A.M. Dirac attended a course of lectures on relativity given by the philosopher Charlie D. Broad, at the time a professor at Bristol. These lectures dealt with the philosophical aspects of relativity, not with the physical and mathematical aspects, which Dirac 
Stéphane Lupasco, Jean-Louis Destouches, André Lamouche, mais également Tullio Levi-Civita ou Paul Langevin). Je m'en tiendrai à l'« intériorité » du texte bachelardien, tout en sachant que rien de tel n'existe réellement ou complètement.

Précédent immédiatement La Valeur inductive de la relativité, il faut se rapporter à tout le chapitre viII de l'Essai sur la connaissance approchée (1928), et au compte rendu de Gaston Rabeau ${ }^{73}$ qui dégage, par anticipation, et de manière précise, les catégories fondamentales (et les auteurs fétiches) qui ne quitteront jamais l'espace du texte bachelardien.

Dans La Valeur inductive de la relativité, les pages 78 à $84^{74}$ et les pages 197 et suivantes sont absolument fondamentales.

L'un des seuls commentateurs de ce texte aura pu noter le sens fondamentalement non standard attribué par Bachelard au concept qui illustre jusqu'à son titre : ainsi, dans son compte rendu des Recherches philosophiques de 1931, Spaier note que « sans prendre soin de nous avertir qu'il ne tient aucun compte de la signification habituelle du mot induction, M. Bachelard qualifie ainsi une multiplication et un enrichissement prémédités de considérations théoriques et même, spécifiquement mathématiques, qui ne s'orientent vers l'expérience que tardivement, par surcroît $^{75}$ ".

Nous allons quant à nous poser de manière abrupte le sens strict de l'origine scientifique d'un concept dont le spectre n'aura de cesse de s'étendre, jusqu'en ses enjeux métaphysiques.

C'est à l'une des sources idéellement reconstructibles, mais en tout cas implicites, de la catégorie bachelardienne que l'on peut renvoyer ici : le dispositif d'induction tel qu'il s'est imposé à Einstein lui-même, et ce depuis sa préoccupation précoce pour l'électromagnétisme. Au cours de l'année 1895, Einstein, alors âgé de seize ans, écrit un texte à l'intention de son oncle César Koch, intitulé «Sur l'examen de l'état de l'éther dans un champ magnétique ${ }^{76}$ ». C'est un texte qu'il savait «naïf et incomplet », notamment parce qu'il ne disposait pas des données qui lui auraient permis

would have preferred. Although he did not appreciate Broad's philosophical outlook, the lectures inspired him to think more deeply about the relationship between space and time. Ever since that time, Dirac was firmly committed to the theory of relativity, with which he soon became better acquainted."

73. G. RABEAU, compte rendu cité supra n. 13.

74. Elles feront l'objet d'un commentaire détaillé dans la suite que je compte donner à cet article.

75. A. SPAIER, compte rendu cité supra n. 14, p. 369.

76. The Collected Papers of Albert Einstein, Princeton, NJ, Princeton University Press, 1987, vol. I : The Early Years, 1879-1902, ed. by John Statchel, D. C. Cassidy, R. SchulMANN, J. ReNN et R. SUMmerfield, p. 6-10. Ce texte est resté longtemps inédit; voir Abraham Pais, Subtle is the Lord. The science and life of Albert Einstein, Oxford, Oxford University Press, 1982, p. 131; J. STATCHEL, "Einstein and Michelson. The context of discovery and the context of justification ", Astronomische Nachschrift, vol. 303, 1, 1982, p. 47-53. 
d'entrer plus profondément dans le sujet : il s'y demandait comment un champ magnétique engendré par un courant affecte l'éther et modifie le courant lui-même, ce qui, en quelque sorte, redécouvrait (sans l'avoir connue) la self-induction. Dans cet essai juvénile, Einstein s'interrogeait sur les propriétés de l'éther élastique en relation au courant électrique et au champ magnétique produit par ce dernier :

«Le cas le plus intéressant, mais aussi le plus subtil, serait l'examen expérimental direct du champ magnétique formé autour d'un courant électrique, parce que l'exploration de l'état élastique de l'éther dans ce cas nous permettrait de regarder la nature énigmatique du courant électrique. »

Ce texte ne fait aucune allusion à James Clerk Maxwell, qu'Einstein ne connaissait probablement pas de façon détaillée. C'est pendant son séjour au Polytechnicum de Zurich, mais toujours par des lectures privées, qu'Einstein étudiera les théories de Maxwell, non enseignées à l'ETH : « À l'époque où j'étais étudiant, le sujet qui me fascinait le plus était sans conteste la théorie de Maxwell ${ }^{77}$."

Et ce sera finalement dans un texte datant de 1912 que s'instaurera le paradigme «inductif » de la Relativité, son titre en donnant déjà l'entière programmatique : «Gibt es eine Gravitationswirkung die der elektrodynamischen Induktionswirkung analog ist $^{78}$ ? » (Existe-t-il une action gravitationnelle analogue à l'induction magnétique?)

Plus encore, l'analogie " inductive », qui correspond à l' « expérience de pensée » opérée sur l'effet réciproque qu'un aimant et un conducteur en mouvement relatif exercent l'un sur l'autre, aura été, de l'aveu même d'Einstein, le motif « inducteur » de sa découverte du « principe de relativité $^{79}$ ». Dans un manuscrit non publié datant de 1920, intitulé « Les idées fondamentales et les méthodes de la théorie de la Relativité exposées selon leur développement ${ }^{80}$ ", Einstein évoque, dans la deuxième partie consa-

77. Voir op. cit. supra n. 19, vol. I, p. 32.

78. Publié dans le Vierteljahresschrift für gerichtliche Medizin, Ser. 3, XLIV, 1912, p. $37-40$.

79. C'est ce même raisonnement qui ouvre l'article fondateur de 1905, "Elektrodynamik bewegter Körper ", Annalen der Physik, Ser. 4, XVII, 1905, p. 891-921, trad. franç. in A. EiNSTEIN, op. cit. supra n. 15, 1993, vol. II, « Relativités I », p. 31 sq. Voir également, in ibid., la note 6 consacrée à August Föppl et à sa discussion, dès 1894, de la relativité du phénomène d'induction dans le cadre de la théorie de Maxwell; August Fôppl, Einführung in die Maxwell'sche Theorie der Elektrizität, Leipzig, Teubner, 1894.

80. «Grundgedanken und Methoden der Relativitätstheorie in ihrer Entwicklung dargestellt $»$, manuscrit original déposé à la Pierpont Morgan Library à New York, connu comme «Manuscrit Morgan ». Sur ce point, voir A. PAïs, Albert Einstein. La vie et l'ceuvre. "Subtil est le Seigneur... », trad. franç. de Christian Jeanmovgin et Hélène SeYrès, Paris, Interéditions, 1993, p. 176 sq. Sur le «filage » analogique dans l'œuvre d'Einstein, voir l'excellente reconstruction de M. PATY, op. cit. supra n. 20, p. 77 sq. 
crée à la Relativité générale, la puissance heuristique renouvelée d'une sorte d'extension inductive de cette première expérience de pensée. C'est elle, en effet, qui lui suggéra en 1907, l'analogie entre la symétrie aimantcircuit électrique, et une symétrie accélération-champ gravitationnel, cette dernière ayant constitué, selon ses propres termes, «die glücklichste Gedanke meines Lebens ». Il reprend, de manière encore plus détaillée, la description donnée dans le paragraphe d'introduction de l'article de 1905 :

«Dans la mise au point de la théorie de la Relativité restreinte, les idées [...] sur l'induction électromagnétique de Faraday [...] ont joué un rôle majeur : selon Faraday, le mouvement relatif d'un aimant par rapport à un circuit électrique crée par induction dans ce dernier un courant électrique. Que ce soit l'aimant ou le conducteur qui bouge, cela est sans importance; c'est seulement une question de mouvement relatif. Mais, suivant la théorie de MaxwellLorentz, l'interprétation théorique de ce phénomène est totalement différente dans un cas et dans l'autre [...]. Cependant, l'idée que deux cas essentiellement différents soient en jeu m'était insupportable. La différence entre les deux, j'en avais la conviction, ne pouvait être qu'une différence dans le choix du point de vue, et non une différence réelle. Si l'on en jugeait du point de vue de l'aimant, il n'y avait à coup sûr aucun champ électrique présent; du point de vue du circuit, un champ électrique se trouvait sûrement présent. L'existence d'un champ électrique était donc relative; elle dépendait de l'état de mouvement du système de coordonnées que l'on utilisait, et ce à quoi l'on pouvait attribuer une sorte de réalité objective, ce ne pouvait être que les champs électrique et magnétique pris ensemble, indépendamment de l'état de mouvement de l'observateur ou du système de coordonnées. Le phénomène de l'induction électromagnétique me poussait à formuler le postulat du principe de relativité ${\text { (restreinte })^{81} . "}^{2}$

«[...] Puis me vint [en 1907] l'idée la plus heureuse de ma vie : le champ gravitationnel n'a qu'une existence relative, à la manière du champ électrique engendré par l'induction magnéto-électrique ${ }^{82}$,

81. Souligné par nous.

82. Sur tout le "fonds " théorique engagé ici, voir le remarquable ouvrage de Françoise Balibar, Einstein 1905. De l'éther aux quanta, Paris, Presses universitaires de France (Philosophies), 1992. En part., sur les questions essentielles des rapports établis entre écriture « algébrique » et écriture " diagrammatique » (le véritable " génie de Faraday ») qui conduiront James Clerk Maxwell à la "production d'une théorie algébrique de l'électromagnétisme ", voir : " De l'importance des diagrammes ", p. 31 sq.; sur la distinction einsteinienne essentielle entre " équations différentielles totales" (discret-matière) et "équations aux dérivées partielles » (continu-champ), et sur sa refonte destinée au départ à gommer une " profonde différence formelle " (équivalence entre masse et énergie), voir " L'éther et l'opposition continu/discontinu », p. 55 sq. et «Unité et substance », p. 120 sq. 
En 1934, renvoyant à un article de Metz sur « La théorie du champ unitaire de M. Einstein ${ }^{83} »$, Bachelard relève à nouveau la centralité relativiste de l'analogie :

« [...] Einstein semble avoir glissé l'idée de relativité juste dans l'intervalle du caractère électrique et du caractère magnétique de l'ancien champ conçu substantivement comme électromagnétique. En effet, en commentant sa nouvelle théorie du champ unitaire il écrit : "Le même état de l'espace qui apparaît comme un champ purement magnétique pour un certain système de coordonnées est, en même temps, pour un autre système en mouvement par rapport au premier, un champ électrique et vice-versa." Cela revient à désigner comme simples apparences les caractères expérimentaux - magnétiques et électriques - qui peuvent être à tour de rôle effacés par des modifications dans la référence géométrique ${ }^{84}$. »

Comme s'il n'était toujours pas sûr d'être entendu sur ce point fondamental, Bachelard renvoie une fois de plus au sens " physique » du terme d'induction, cette fois dans un texte datant de 1942, et publié dans la Revue philosophique de la France et de l'étranger ${ }^{85}$. Mais, apparent paradoxe, il s'agit ici d'un texte non strictement épistémologique intitulé : «Une psy-

83. Revue philosophique, nov. 1929 , p. 393. L'article cité d'EINSTEIN, « The new field theory », fut publié dans le Times de Londres daté du 4 février 1929; il devait être suivi d'un second article le lendemain dans les colonnes du même quotidien. Ces deux textes sont la version simplifiée de sa théorie du champ unitaire communiquée à l'Académie des sciences de Berlin : «Riemann-Geometrie mit Aufrechterhaltung des Begriffes des Fernparallelismus », Sitzungsberichte der Preussische Akademie der Wissenschaften, Phys.-Math. Klasse, Berlin, 1928 (séance du 7 juin); « Neue Möglichkeit für eine einheitliche Feldtheorie von Gravitation und Elektrizität », ibid. (séance du 14 juin); et «Zur einheitlichen Feldtheorie », ibid., 1929 (séance du 8 janvier).

84. G. Bachelard, Le Nouvel Esprit scientifique, Paris, Alcan, 1934, ici, Paris, Presses universitaires de France, $1968^{10}$, p. 125.

85. Revue philosophique de la France et de l'étranger, 132, 1942-1943. Notons que c'est très précisément dans un contexte relativiste strict qu'en 1928 Tullio Levi-Civita associe déjà, et très précisément, « constructivisme ", « justification inductive " et notions de " première et seconde approximations " à propos de la solidarité des phénomènes physiques dans les équations gravitationnelles de la Relativité générale. Ainsi, le § 3, chap. n, «Équations gravitationnelles et Relativité générale », s'intitule : «Solidarité des phénomènes physiques. Critère constructif des équations gravitationnelles et réduction de leur justification inductive au cas statique », in Tullio LevI-Civita, Fondamenti di meccanica relativistica, redatti dal prof. E. Persico, Bologne, Zanichelli, 1928, p. 96. Rappelons que T. Levi-Civita (Padoue, 1873Rome, 1941), élève de Gregorio Ricci-Cubastro (Lugo, 1853-Bologne, 1925) à l'université de Padoue, élabora avec son maître l'analyse tensorielle : voir l'ouvrage canonique paru en 1901, publié d'abord en français, intitulé Méthodes de calcul différentiel absolu et leurs applications. En 1917, il introduit en géométrie différentielle le concept de « déplacement parallèle " dans les espaces à courbure quelconque qui connâtra des applications immédiates en Relativité, et dans les tentatives d'unification des théories de la gravitation et de l'électromagnétisme. Pour une analyse globale de cette ouvre fondamentale, voir Tullio Levi-Civita. Convegno internazionale celebrativo del centenario della nascita. Atti dei Convegni dei Lincei, Roma, 17-19 dic. 1973, Rome, Accademia dei Lincei, 1975. 
chologie du langage littéraire : Jean Paulhan, "Les Fleurs de Tarbes ou la Terreur dans les lettres" ${ }^{86}$. $»$ Le voici, en son contexte initial :

« [...] Les critiques portent souvent plus d'attention au mot qu'à la phrase - à la locution plus qu'à la page. Ils pratiquent un jugement essentiellement atomique et statique. Rares sont les critiques qui essaient un nouveau style en se soumettant à son induction. J'imagine, en effet, que de l'auteur au lecteur devrait jouer une induction verbale qui a bien des caractères de l'induction électromagnétique entre deux circuits. Un livre serait alors un appareil d'induction psychique qui devrait provoquer chez le lecteur des tentations d'expression originale ${ }^{87}$. $»$

Ce philosophème sera repris (entre autres) en 1953 dans «Germe et raison dans la poésie de Paul Éluard ${ }^{88} »$ :

«Les poèmes d'Éluard sont des diagrammes de confiance, des modèles de dynamisation psychique [...]. Si l'on est sensible à l'induction psychique d'éveil, de réveil - de naissance, de rénovation - , de jeunesse et de jouvence, on ne s'étonnera pas de la puissance vraie des poèmes réunis sous le signe du Phénix. Ici encore, nous recevons le bienfait d'une condensation de forces exceptionnelles. »

Nous noterons ici, sans plus de commentaires, la (re)mobilisation de la chaîne catégoriale «dynamisation ", « induction », « condensation » et " diagramme $"{ }^{89} \ldots$

Si nous insistons si lourdement sur cet usage têtu du modèle électromagnétique de l'induction, c'est parce que nous pensons que les méprises

86. Sur le prétendu versant poétique nocturne de Bachelard, démon sans lien avec son activité épistémologique, deux citations entre cent. Tout d'abord, sur l'« isomorphisme » de ces deux fonctions ou de ces deux domaines, voir François Dagognet, Gaston Bachelard. Sa vie, son auvre, Paris, Presses universitaires de France, 1965, p. 56 : « Aucun doute n'est permis, science et poésie, toutes deux spécifiquement ontogéniques, dépassent et renouvellent le monde, lui substituent une matière nouménale, relèvent d'une philosophie de l'énergie » ; voir également Lautréamont, op. cit. supra n. 5, p. 70-71: «Quels sont les éléments d'une forme géométrique qui peuvent être impunément déformés dans une projection en laissant subsister une cohérence géométrique? [théorème fondamental de la géométrie projective] "; " Quels sont les éléments d'une forme poétique qui peuvent être impunément déformés par une métaphore, en laissant subsister une cohérence poétique? [théorème fondamental de la poésie projective] [...] La déformation des images doit alors désigner d'une manière strictement mathématique, le groupe des métaphores. "

87. G. Bachelard, Du droit de rêver, Paris, Presses universitaires de France, 1970, ici $1988^{6}$, p. 181.

88. Voir la revue Europe, 93, 1953.

89. Pour une approche contemporaine des plus affines, voir Alain CONNEs, «À la recherche d'espaces conjugués ", in Ilke Angela Maréchal, Sciences et imaginaire, Paris, Albin Michel, 1998 (nous soulignons) : «Ainsi, pour moi, la représentation mentale algébrique a les mêmes ingrédients, à la fois linguistique et musique, que la poésie [...]. La réalité mathématique brute a une nature inductive. L'activité du mathématicien la comprend de manière projective. » Sur ce topos projectif, voir supra n. 87. 
logico-empiristes sur son sens et ses sources sont la cause des mésinterprétations et du mépris suscités par ce livre qui va même jusqu'à en inscrire l'essentialité au lustre même de son titre. «Induction» semble toujours avoir été compris comme le contraire même de ce qu'est son essence bachelardienne pourtant explicitement revendiquée. Il ne s'agit en aucun cas du sens restreint d'induction logique ou d'inférence logique. C'est d'ailleurs cette même induction logique qu'Einstein réfutait régulièrement, au profit de la construction de concepts ${ }^{90}$. Bachelard était tellement conscient du retournement possible, contre lui, de ses propres positions, qu'il pointa même la raison de cette aberration perverse dans sa marque d'une réserve d'emploi étonnante, aussi bien en son contexte qu'en son conteste :

« Pour bien comprendre les nuances diverses de cette sublimation active et en particulier la différence radicale entre la sublimation cinématique et la sublimation vraiment dynamique, il faut se rendre compte que le mouvement livré par la vue n'est pas dynamisé. Le mobilisme visuel reste purement cinématique. La vue suit trop gratuitement le mouvement pour nous apprendre à le vivre intégralement, intérieurement. Les jeux de l'imagination formelle, les intuitions qui achèvent les images visuelles nous orientent à l'envers de la participation substantielle. Seule une sympathie pour une matière peut déterminer une participation réellement active qu'on appellerait volontiers une induction si le mot n'était déjà pris dans la psychologie du raisonnement. Ce serait pourtant dans la vie des images que l'on pourrait éprouver une volonté de conduire. Seule cette induction matérielle et dynamique, cette " duction " par l'intimité $\mathrm{du}$ réel, peut soulever notre être intime. Nous l'apprendrons en établissant entre les choses et nous-mêmes une correspondance de matérialitế ${ }^{91}$."

Est-ce à nouveau si étonnant qu'un essai du versant de l'Euvre dite littéraire, qualifiée également de face "nocturne ", définisse le champ, boucle la boucle (magnétique et matérielle) de la métaphore sur tout un courant discursif, et au fil du raisonnement? C'est pourtant bien la mise en œuvre du nerf à la fois "spectral » et secret de toute l'argumentation bachelardienne et, a fortiori, einsteinienne qui s'exhibe ici, devant nous. Le théâtre des opérations, toujours habité du «spectre » exemplaire du geste einsteinien, est sans cesse (et fantomatiquement) réinvesti comme champ, mais en le nommant, cette fois, et comme tel, par antonomase, autoréférence, déplacement transférentiel et métonymie. C'est ici la clé de l'imagination du mouvement, comme mouvement de l'imagination scientifico-poḯtique, qui se donne à voir :

90. Voir, par ex. (et entre autres textes), A. EinsTEIN, «Induction et déduction en physique ", article publié dans le quotidien à grande diffusion Berliner Tagesblatt, le 25 décembre 1919 , repr. in op. cit. supra n. 15, 1991, vol. V, « Science, éthique, philosophie », p. 94-96.

91. G. Bachelard, L'Air et les songes. Essai sur l'imagination du mouvement, Paris, José Corti, 1943, p. 15. 
a) par la mise en évidence du "petit laboratoire " électromagnétique inductif d'une nouvelle révolution scientifique. L'appareil expérimental, chargé de théorie, est à nouveau frais habité en mode heuristique et expérimental, source première d'une expérience de pensée. Il est ce qui montre et ce qui formule l'avancée relativiste, son architecture ${ }^{92}$.

ß) par le rabattement du dispositif inductif réel sur les conditions de sa «pensée », modèle de toute pensée (scientifique, philosophique et/ou poétique). Comme dans le texte du très jeune Einstein, cette induction recèle et révèle son essence de self-induction. «Source » matérielle, métaphorique, et « champ " thématique sont self-consistant. Par une sorte d'invagination du dispositif expérimental et du dispositif théorique, Bachelard induit, en révélant par là le secret et en en rendant visible le "spectre ", son propre dispositif philosophique. S'opère alors un véritable autodéveloppement (au sens photographique strict des termes, mouvement lumineux réel à même son inscription symbolique) où l'opérateur matériel et expérimental se montre comme opérateur spéculatif auto-adjoint : «Un élément matériel est le principe d'un bon conducteur qui donne la continuité à un psychisme imaginant ${ }^{93}$. $\gg$

L'enjeu profond est celui d'une sorte de parallélisme des attributs de la pensée et de l'étendue, de la théorie et du réel chiasmatiquement autoinduits, où le mouvement livre sa dynamique. Dès lors, « seule cette " duction " par l'intimité du réel, peut soulever notre être intime "; et c'est ce qui donne toute sa force à l'impératif de Painlevé nous enjoignant d'habiter le secret des relativistes qui firent cercle autour d'Einstein : « Regardez, on les voit se comprendre."

Pour le relativiste, " [établir] entre les choses et nous-mêmes une correspondance de matérialité » ne peut être autre chose que le geste infiniment inducteur d'auto-application à la doctrine relativiste de son propre principe ${ }^{94}$. C'est bel et bien prendre la relativité au mot et la mettre « dans tous ses états ».

92. Voir ici le texte admirable d'un auditeur d'exception (seul « littéraire ") présent à la conférence d'Einstein tenue à l'institut Henri Poincaré le mardi 12 novembre 1929, à Paris : Paul VALÉRY, L'Idée fixe, Paris, Gallimard, 1934, ici $1961^{2}$, p. 143 : « La distance entre la théorie et l'expérience est telle qu'il faut bien trouver des points de vue d'architecture. "In ID., Cahiers, II, Paris, Gallimard (Bibliothèque de la Pléiade), p. 875, on peut lire également : « $129^{\text {bre }}$, à 5 h 30 . Conf[érence] d'Einstein. Je suis très intéressé vers la fín. Il se montre un grand artiste. " Les deux conférences d'Einstein furent l'occasion d'un contact personnel avec Elie Cartan. Les textes des deux conférences (des 8 et 12 nov.) furent rédigés par Alexandre Proca et publiés dans le premier fascicule des Annales de l'IHP, Paris, 1930, sous le titre "Théorie unitaire du champ physique ».

93. G. BaCHELARD, op. cit. supra n. 91, p. 14.

94. Sur cet enjeu, voir L. NotTale, op. cit. supra n. 62, et dans ce numéro, p. 165-174, la revue critique de Joël Merker sur cet ouvrage. Est-ce un hasard si c'est au philosophe Maurice Solovine qu'Einstein se confiera? Voir A. EINSTEIN, Lettres à Maurice Solovine/Briefe an Maurice Solovine, Paris, Gauthier-Villars, 1956, lettre du 30 octobre 1924 : «Mon intérêt 
Accompagnant ce noyau de sens technique, devenu originaire par récurrence épistémologique, on devra parler d'une véritable induction méta-physique appelée philosophiquement de ses vœux par Bachelard, et ce dès l'Essai sur la connaissance approchée.

Ce qui fait la vraie nouveauté des théories nouvelles est pour Bachelard d'ordre méta-physique et spéculatif, c'est-à-dire attaché à de l'événement de pensée. Que ce soit à propos de la Relativité (La Valeur inductive de la relativité) ou de la Mécanique ondulatoire (L'Activité rationaliste de la physique contemporaine), il considère que ces théories introduisent une rupture, non seulement dans l'histoire de la physique, mais dans l'ordre de l'explication : elles obligent à penser autrement, et pas simplement autre chose. Cette rupture s'introduit par conséquent dans la signification ou la portée de la démarche scientifique.

Dans le même temps, ce qui explique notre déroute face à ces nouvelles théories réside dans un déficit de la pensée, et donc de la pensée métaphysique. Ici les exemples sont légions; ils sont l'exemplarité même de tous les exemples bachelardiens du retard philosophique. L'exemple est pris dans un texte de 1932 :

« [À propos de l'organisation quantique du plan de Mendéléeff : on] pourrait aussi objecter que la règle de Bohr garde un certain arbitraire et qu'elle retrouve d'une manière factice la correspondance du nombre des électrons avec le nombre des éléments dans les diverses périodes. On ne voit pas bien par quels caractères l'électron fixe les quanta. Mais c’est là encore la fausse idée réaliste de la qualification directe de l'électron par les quanta qui amène cette objection. Nous souffrons d'un " déficit" de la pensée métaphysique. En effet, il paraît bien nous manquer encore un type de pensée qui expliquerait, par une sorte d'attribution de groupe, le partage des quanta sur les différents électrons. Autrement dit, la pluralité des électrons et la pluralité des quanta devraient être mises en correspondance immédiate. C'est dans cette voie qu'on pourrait peutêtre interpréter directement la règle de $\mathrm{Pauli}^{95}$. »

Contrairement à ce qu'avait imposé, à la fin des années 1960, la vulgate bachelardienne (ou pseudo-bachelardienne), il nous faut affirmer haut et

pour la science s'est toujours cantonné à l'étude des principes, et c'est cela qui explique le mieux ma conduite »; il parle également du "désir ardent de la saisie des principes ».

95. G. Bachelard, Le Pluralisme cohérent de la chimie moderne, Paris, Vrin, 1932; à propos de la «description quantique ", p. 220-221. 
fort que le sens de cette réflexion sur la science est celui d'un immense protreptique, une défense et illustration, mieux, une exhortation à philosopher à travers l'impulsion que l'«esprit » scientifigue donne à-l'esprit lout court. Parce qu'il porte la pensée à son activation maximale, l'objet scientifique possède une éclatante supériorité d'instruction métaphysique sur l'objet d' "expérience commune ${ }^{96}$ ", et oblige le philosophe à pénétrer dans « des zones d'extrême sensibilité philosophique ", alors qu'inversement, les «simplifications philosophiques [l'effacement des nuances le long de pensées appauvries] privent précisément la pensée philosophique d'un extraordinaire exercice d'assouplissement ${ }^{97}$ ". " Les mathématiciens nous ont appris à totaliser les formes d'espace et de temps dans un espacetemps. Les métaphysiciens, plus timides que les mathématiciens, n'ont pas tenté la synthèse métaphysique correspondante ${ }^{98}$. $\gg$

Sur ce plan, et nous y retrouvons à nouveau la signature exemplaire d'Einstein, Bachelard multipliera de plus en plus souvent dans ses derniers textes les interpellations ad philosophum. Ainsi, cette exhortation formulée en 1949, dans une langue dite universelle, pour ne pas dire impériale (du moins pour la science contemporaine), l'anglais :

«Les vertus philosophiques de la révolution einsteinienne, comparées aux métaphores philosophiques de la révolution copernicienne, auraient une tout autre efficacité si seulement les philosophes voulaient rechercher toutes les raisons d'enseignement de la science relativiste [...]. La science éprouve alors ce que Nietzsche appelle "un tremblement de concepts", comme si la Terre, le Monde, les choses prexaieat une autre structure du fait qu'on pose l'explication sur de nouvelles bases ${ }^{\$ 9}$."

Einstein est ici plus qu'un physicien génial, c'est un électrochoc métaphysique à l'usage des philosophes.

Insister sur l'enjeu métaphysique du travail bachelardien n'est pas ici seulement de l'ordre de la provocation.

Il est impossible de séparer l'usage que Bachelard fait du mot "métaphysique " de la prise en compte d'un jeu de préfixes portant sur quatre index principaux: méta-, pan-, non- et sur-. Sans être exactement synonymes, tous appartiennent à une même orientation synthétique et dỉalectique, sont porteurs de la même "valeur inductive".

96. ID., op. cit. supra n. 69 , p. 53 .

97. ID., op. cit. supra n. 68 , ici $1965^{2}$, p. 182.

98. ID., La Philosophie du non. Essai d'une philosophie du nouvel esprit scientifique, Paris, Presses universitaires de France, 1940, ici $1966^{4}$, p. 67.

99. ID., "The philosophical dialectic of the concepts of relativity $"$, in op. cit. supra n. 19, repr. dans la version française citée supra n. 30, p. 120. 
Mais le terme "métaphysique " pose un problème particulier chez un auteur dont la référence centrale est la physique. Il existe chez lui deux types de parallélisme d'abord déconcertants.

\section{«Métaphysique »/« Métachimie »}

Sur cette question, il faut renvoyer à La Philosophie du non (1940) et à sa «dialectisation" de la notion de substance. Bachelard y définit d'ailleurs sa «fonction plus proprement philosophique » de la façon suivante : «[...] essayer d'atteindre la zone où l'esprit pense en hésitant, où il se risque hors de sa propre expérience, où il s'offre avec une tranquille imprudence à toutes les polémiques ${ }^{100}$." 1940, en France, fut donc une époque où il y avait encore de la pensée, de l'idée, et donc de la résistance, puisqu'il subsistait encore de la prise de risque et du polemos : car, comme le clame si justement Badiou citant Mao Tsê-Tung: "Si vous avez une idée, il faudra qu'un se divise en deux ", ce qui ne peut plus être le cas de l'éthique libéralo-totalitaire, pur supplément d'âme d'un consensus à qui la «division en deux » fait tant horreur.

Voici comment se définit la «métachimie »:

" Dès maintenant, une métachimie nous paraît possible. Si on pouvait la développer, cette métachimie devrait disperser le substantialisme [...]. La métachimie serait à la métaphysique, dans le même rapport que la chimie à la physique. La métaphysique ne pouvait avoir qu'une notion de substance, parce que la conception élémentaire des phénomènes physiques se contentait d'étudier un solide géométrique caractérisé par des propriétés générales ${ }^{101}$. »

\section{"Métaphysique »/ Métamicrophysique "}

Cette distinction semble à première vue ne reposer que sur le découpage de deux âges scientifiques ou de deux niveaux d'expérimentation. Encore timide en 1934 dans l'« Introduction" au Nouvel Esprit scientifique, où, après avoir proposé «une pédagogie de l'ambiguitté » et posé l'idée « d'établir [en d'autres lieux] la métaphysique qui doit servir de base à la Physique moderne", Bachelard veut "fonder une ontologie du complémentaire ${ }^{102}$. Près de vingt ans plus tard, il va même jusqu'à déclarer que la microphysique occupe dans la topologie des notions objectives, la place et la fonction de la chose en soi, ou plutôt, qu'elle réalise la synthèse entre la notion négative de chose en soi, et celle, positive, de noumène ${ }^{103}$.

100. ID., op. cit. supra n. 98, p. 93.

101. Ibid., p. 53.

102. G. BACHELARD, op. cit. supra n. $84,1968^{10}$, p. 15-16.

103. ID., op. cit. supra n. 68. Voir aussi, ID., op. cit. supra n. 69, p. 110 : « Pour caractériser pleinement un objet qui réalise une conquête théorique de la science, il faudrait parler d'un noumène nougonal, d'une essence de pensée qui engendre des pensées. " Je n'insiste pas ici sur le sens profond de ces reprises catégoriales déplacées, c'est-à-dire réélaborées, et dont le projet philosophique sous-jacent est la construction d'un non-kantisme. Le choix du kantisme 
En dépit d'une floraison de constructions verbales quasi inépuisable (cf. «métamécanique », «métapsychologie », «métapoétique »), Bachelard n'aura jamais recours au terme de "métamathématique », c'est-à-dire au seul composé orthodoxe reçu dans la littérature scientifique, alors qu'il emploie couramment les termes de "pangéométrie ", "panarithmétique ", etc.

On peut avancer une raison négative toute prête, accordée au rejet du programme logiciste (notamment hilbertien) habituellement associé au terme, et à une vision antipositiviste (ou antinominaliste) des mathématiques ${ }^{104}$.

Mais cette «absence » calculée présente sa face positive dans le fait que les mathématiques sont déjà animées d'une visée métaphysique. Le symbole par excellence de leus nouvelle fonction est, d'une part, celui des tenseurs (ce que nous verrons en détail dans un prochain volet de ces analyses, quand nous tenterons de jouer les prolongations «actuelles » du programme bachelardien [en particulier, sur le " principe de la conservation des formes algébriques » et la «spectralité tensorielle»], de Tullio LeviCivita à Roger Penrose et John Archibald Wheeler ${ }^{105}$ ). Un formulaire

- en part. in La Philosophie du non -, comme base métaphysique, s'explique évidemment par la décision de subordonner l'objectivité du réel à la discursivité de la méthode; c'est le rappel fréquent, sous la base dualistique de la philosophie scientifíque, du privilège métaphysique de la perspective allant de la rationalité à l'expénence ratjonnellement construite. Voir G. BACHELARD, op. cit. supra n. 98, p. 6: «Nous interpréterons donc, dans le sens d'un rationalisme, la suprématie toute récente de la Physique mathérmatique [...]. La réalisation d'un programme rationnel d'expériences détermine une réalité expérimentale sans irrationalité. " Un autre criticisme est alors réclamé, le «criticisme relativiste », voir ID., L'Engagement rationaliste, op. cit. supra n. 30, p. 124 : " La double philosophie de l'expérience de l'espace - la philosophie réaliste et la philosophie kantienne - doit être remplacée par une philosophie dialectique de l'espace, par une philosophie à la fois expérimentale et rationnelle. En somme, dans la Relativité, la philosophie de l'expérience ultra-fine et la philosophie de la théorie physique sont fortement couplées. La philosophie de la science va se révéler comme une philosophie critique plus liante, plus synthétique que ne le fut la philosophie kantienne à l'égard de la science newtonienne. Le criticisme relativiste ne se borne pas à une révolution des privilèges d'explication. Il est plus profondément révolutionnaire. Il est plus génial. » Ainsi, le nouvel esprit aura les caractéristiques d'un « kantisme de deuxième espèce », kantisme fonctionnel animé d'un « tremblement nietzschéen » des concepts fondamentaux.

104. Sur ce point, voir dans ce muméro, p. 111-138, l'aricle de Giuseppe LoNGO, "L'intelligence mathématique, l'infini et les machines », dont les raisons de rejet peuvent être considérées comme affines.

105. En part., dans l'article de Gregorio Ricci et Tullio Levi-CrvTtA, « Méthode de calcul différentiel absolu et leurs applications », Mathematische Annalen, 54, 1901, p. 125-201; Élie CARTAN, « Les systèmes différentiels extérieurs et leurs applications géométriques », Exposés de Géométrie 14, Paris, Hermann, 1945; ID., Differential Forms, Paris, Hermann, 1970; J. A. Schouten, Ricci-Calculus, Berlin, Springer, 1924, 1954² ; Charles W. Misner, Kip S. Thorne, John Archibald Wheeler, Gravitation, San Francisco, W. H. Freeman and Co., 1973; Roger Penrose et Wolfgang Rindler, Spinors and space-time, Cambridge, Cambridge University Press, 1984. 
particulièrement dense ramasse cette nouvelle fonction, à la fois symbolique et métaphysique, assumée par le tenseur :

«Avant l'ère mathématique, durant l'âge du solide, il fallait que le Réel désignât au physicien, dans une prodigalité d'exemples, l'idée à généraliser : la pensée était alors un résumé d'expériences accomplies. Dans la nouvelle science relativiste, un unique symbole mathématique, dont la signification est prolixe, désigne les mille traits d'une Réalité cachée : la pensée est un programme d'expériences à réaliser ${ }^{106}$. "

L'autre symbole est celui des opérateurs que Bachelard associe à une inversion philosophique décisive touchant la perspective de l'objectivité ${ }^{107}$.

Deux derniers exemples, parmi tant d'autres, vont être pris ici pour «typique » et comme «topique » :

1) la "relativation ${ }^{108}$ " de Born, et la théorie dite fondamentale d'Eddington ${ }^{109}$;

106. Rappelons, en forme d'insistance, deux occurrences sur le même thème, où la visée bachelardienne de la «spectralité » tensorielle et de la puissance d' « induction formelle » ou « algébrique » vient converger avec l'approche de l'initiateur des études relativistes en France, voir G. BACHELARD, op. cit. supra n. $84,1968^{10}$, p. 54 : «Il y a quelques années, M. Langevin nous disait: "Le Calcul Tensoriel sait mieux la physique que le Physicien lui-même." Le Calcul Tensoriel est vraiment le cadre psychologique de la pensée relativiste. C'est un instrument mathématique qui crée la science physique contemporaine comme le microscope crée la microbiologie. Pas de connaissances nouvelles sans la maîtrise de cet instrument mathématique nouveau. » Plus remarquable encore, cette confirmation extensive, quatre ans plus tard, de la part de P. LANGEvin, New Theories in physics, op. cit. supra n. 63, p. 230 : «In fact, the formalism of our theories knows more Physics than we do, and we can trust it. It is also certain that Dirac's formalism, by making the equation of electro-magnetism linear, was a sure guide to Dirac. »

107. Voir G. Bachelard, L'Expérience de l'espace dans la physique contemporaine, Paris, Alcan, 1937, chap. IV, « Les opérateurs mathématiques », et ID., op. cit. supra n. 68, chap. vII, «Les opérateurs ».

108. Le syntagme "relativation " a été forgé par Maurice Solovine dans sa traduction d'Einstein, L'Éther et la théorie de la Relativité, Paris, Gauthier-Villars, 1921, p. 13, note, pour traduire le mot allemand Relativierung, sans équivalent dans la langue française. Voir G. BACHELARD, op. cit. supra n. 7, p. 100, et M.-A. Tonnelat, op. cit. supra n. 9, p. 238, n. 1 : « Max Born utilise le mot dans un sens plus large correspondant à un renoncement croissant aux descriptions subjectives : la "relativation " de la notion de force commence, pour lui, dès l'abandon de l'idée d'effort au profit de celle de poids, puis de pesanteur, et enfin de gravitation universelle.

Pour nous, la relativation, processus spécifiquement relativiste, consiste non pas à renoncer au particulier pour le général mais, plus précisément, à regrouper des notions que la référence avait dispersées. Elle exige la reconnaissance d'une structure invariante intrinsèque, autrement dit, d'une " réalité objective". "

109. M. BorN, La Théorie de la Relativité d'Einstein et ses bases physiques, trad. franç. de F.-A. Finkelstein et J.-G. Verdier, Paris, Gauthier-Villars, 1923, p. 282-283 (voir supra n. 23). Sur ce point, voir G. BACHELARD, op. cit. supra n. 7, p. 152 : «Cette curieuse équation qui symbolise une relation de symboles : $3 \times 10^{10}$ centimètres $=\sqrt{-} 1$ seconde [la vitesse fondamentale de la lumière comme "pièce d'une construction algébrique "], Minkowski l'appelle, sans doute ironiquement, l'équation mystique. Seul un effort supra-rationnel peut réunir dans une même pensée des entités séparées par une hétérogénéité si crue qu'elle peut passer pour métaphysique. "Sur la « théorie fondamentale » d'Eddington, voir N.-B. SLATER, 
2) la physique topologique et l'espace corpusculaire de Jean-Louis Destouches, qui venait de créer, aux yeux de Bachelard, «la Métaphysique de la science " :

"En fait, les travaux de M. Jean-Louis Destouches réalisent une sorte de panontologie abstraite. Dans sa thèse ${ }^{10}$, il a pu réunir en un seul ensemble cohérent les principes d'une mécanique ponctuelle générale, puis ceux d'une mécanique ondulatoire générale. En étudiant les connexions de ces deux grandes organisations théoriques, $M$. Destouches en arrive à la conception la plus générale susceptible d'englober la pensée scientifique de notre temps. Il propose d'appeler cette conception physique générale la Métamécanique. Un autre nom lui conviendrait mieux, si Aristote et tous les philosophes n'étaient passés par là et n'avaient créé le mot vague avant de posséder la chose précise. C'est, en effet, dans le sens le plus précis du terme, la Métaphysique de la science contemporaine que vient de créer M. Jean-Louis Destouches. Cette métaphysique pourra être étendue, perfectionnée, absorbée par une construction plus générale. Elle ne pourra plus être supprimée, car cette métaphysique est une mathématique, et elle doit bénéficier de la pérennité de la pensée mathématique. Il n'y a qu'une manière de la contredire partiellement : c'est de l'achever ${ }^{111}$. »

Dans ces deux cas, Bachelard retrouve la même notion d'une sorte de deuxième révolution, de révolution au sein de la « révolution copernicienne " kantienne. Pour lui, Eddington effectue la révolution (einsteinienne) de l'idéalisme; Destouches produit celle (quantique) de l'abstraction: «[...] une révolution copernicienne de l'abstraction doit être tentée ${ }^{112}$.»

Charles Alunn

(février 1999).

The Development and meaning of Eddington's « Fundamental Theory ", Cambridge, Cambridge University Press, 1957.

110. Il s'agit des Principes fondamentaux de physique théorique, préf. de Louis DE BRoGLIE, Paris, Hermann, 1942.

111. G. BACHELARD, L'Expérience de l'espace dans la physique contemporaine, op. cit. supra n. 107, p. 136-137.

112. Ibid., conclusion, p. 139. 